\title{
ASYMPTOTICS FOR CRITICAL NONCONVECTIVE TYPE EQUATIONS
}

\author{
NAKAO HAYASHI, ELENA I. KAIKINA, and PAVEL I. NAUMKIN
}

Received 18 March 2003

\begin{abstract}
We study large-time asymptotic behavior of solutions to the Cauchy problem for a model of nonlinear dissipative evolution equation. The linear part is a pseudodifferential operator and the nonlinearity is a cubic pseudodifferential operator defined by means of the inverse Fourier transformation and represented by bilinear and trilinear forms with respect to the direct Fourier transform of the dependent variable. We consider nonconvective type nonlinearity, that is, we suppose that the total mass of the nonlinear term does not vanish. We consider the initial data, which have a nonzero total mass and belong to the weighted Sobolev space with a sufficiently small norm. Then we give the main term of the large-time asymptotics of solutions in the critical case. The time decay rate have an additional logarithmic correction in comparison with the corresponding linear case.
\end{abstract}

2000 Mathematics Subject Classification: 35Q35.

1. Introduction. We study large-time asymptotics of solutions to the Cauchy problem for dissipative equations

$$
\begin{gathered}
u_{t}+\mathcal{N}(u)+\mathscr{L} u=0, \quad t>0, \\
u(0, x)=u_{0}(x), \quad x \in \mathbb{R} .
\end{gathered}
$$

The linear part of (1.1) is a pseudodifferential operator defined by the Fourier transformation

$$
\mathscr{L} u=\overline{\mathscr{F}} \xi \rightarrow x(L(\xi) \hat{u}(\xi)),
$$

and the nonlinearity $\mathcal{N}(u)$ is a cubic pseudodifferential operator of nonconvective type:

$$
\begin{aligned}
\mathcal{N}(u)= & \overline{\mathscr{F}} \xi \rightarrow x \int_{\mathbb{R}} a(t, \xi, y) \hat{u}(t, \xi-y) \hat{u}(t, y) d y \\
& +\overline{\mathscr{F}} \xi \rightarrow x \int_{\mathbb{R}^{2}} b(t, \xi, y, z) \hat{u}(t, \xi-y) \hat{u}(t, y-z) \hat{u}(t, z) d y d z,
\end{aligned}
$$

defined by the symbols $a(t, \xi, y)$ and $b(t, \xi, y, z)$. Consider here the real-valued solution $u(t, x)$. The direct Fourier transformation $\mathscr{F}_{x \rightarrow \xi}$ is

$$
\widehat{u}(\xi) \equiv \mathscr{F}_{x \rightarrow \xi} u=(2 \pi)^{-1 / 2} \int_{\mathbb{R}} e^{-i \xi x} u(x) d x
$$

and the inverse Fourier transformation $\overline{\mathscr{F}_{\xi}} \rightarrow x$ is

$$
\check{u}(x) \equiv \overline{\mathscr{F}} \xi \rightarrow x=(2 \pi)^{-1 / 2} \int_{\mathbb{R}} e^{i \xi x} u(\xi) d \xi .
$$


We suppose that the symbols $a(t, \xi, y)$ and $b(t, \xi, y, z)$ are continuous functions with respect to time $t>0$ and the operators $\mathcal{N}$ and $\mathscr{L}$ have a finite order, that is, the symbols $a(t, \xi, y), b(t, \xi, y, z)$, and $L(\xi)$ grow with respect to $\xi, y$, and $z$ no faster than a power of some order $\kappa$ :

$$
\begin{gathered}
|L(\xi)| \leq C\langle\xi\rangle^{\kappa}, \quad|a(t, \xi, y)| \leq C\left(\langle\xi\rangle^{\kappa}+\langle y\rangle^{\kappa}\right), \\
|b(t, \xi, y, z)| \leq C\left(\langle\xi\rangle^{\kappa}+\langle y\rangle^{\kappa}+\langle z\rangle^{\kappa}\right) .
\end{gathered}
$$

Here and below, we denote by $C$ different positive constants $\langle\xi\rangle=\sqrt{1+\xi^{2}}$.

Model equations (1.1) contain many well-known equations of modern mathematical physics and describe various wave processes in different media. For example, when $\mathcal{N}(u)=u^{3}$ and $\mathscr{L} u=-u_{x x}$, that is, $a(t, \xi, y)=0, b(t, \xi, y, z)=1$, and $L(\xi)=\xi^{2},(1.1)$ transforms into the cubic nonlinear heat equation

$$
u_{t}+u^{3}-u_{x x}=0, \quad x \in \mathbb{R}, t>0 .
$$

Another example is the potential Whitham equation

$$
u_{t}+\left(u_{x}\right)^{2}+u_{x x x}+\mathscr{H} u_{x x x}=0, \quad x \in \mathbb{R}, t>0,
$$

which follows from (1.1) if we take $\mathcal{N}(u)=\left(u_{x}\right)^{2}$ and $\mathscr{L} u=u_{x x x}+\mathscr{H} u_{x x x}$, that is, $a(t, \xi, y)=-(\xi-y) y, b(t, \xi, y, z)=0$, and $L(\xi)=|\xi|^{3}-i \xi^{3}$, where $\mathscr{H}(\phi)=\operatorname{PV}(1 /$ $\pi) \int(\phi(y) /(x-y)) d y$ is the Hilbert transformation. Equation (1.8) comes from the Whitham [21] equation

$$
v_{t}+v v_{x}+v_{x x x}+\mathscr{H} v_{x x x}=0, \quad x \in \mathbb{R}, t>0,
$$

when we introduce a potential $u=\int_{-\infty}^{x} u(t, x) d x$, which vanishes as $x \rightarrow \infty$ if we consider initial data $v(0, x)$ with zero total mass $\int_{\mathbb{R}} v(0, x) d x=0$, therefore $\int_{\mathbb{R}} v(t, x) d x=$ 0 for all $t>0$ in view of (1.9).

Large-time asymptotics of solutions to the Cauchy problems for nonlinear evolution equations was extensively studied. A great success in the study of the large-time asymptotics first of all was achieved by inverse scattering transform method (see [1, 3]). In the super critical case, large-time asymptotics of solutions is similar to that for the linearized case (see $[2,4,18,20]$ and the references cited therein). Critical and subcritical dissipative equations with nonconvective type nonlinearities were considered in papers $[5,6,7,8,9,10,11,12,13,14,15,16,17,19,22]$.

In $[7,9]$, the Cauchy problem of the cubic nonlinear heat equation (1.7) was considered and the time decay estimate

$$
\|u(t)\|_{L^{\infty}} \leq C t^{-1 / 2}(\log \langle t\rangle)^{-1 / 2}
$$

was shown. This result was extended to the case of the porous media equation with critical exponents (see [8]). On the other hand, the asymptotic behavior in the time of the positive solutions to the equation

$$
u_{t}-\Delta u=-u^{p}, \quad x \in \mathbb{R}^{n}, t>0
$$


was studied in $[5,6,7,9,18,19]$. In particular, in [7, 9], it was shown that if the initial data are not negative and $p=1+2 / n$, then the solution decays in time as $(t \log t)^{-n / 2}$ for any $x \in \mathbb{R}^{n}$. It is the $\log t$ behavior that is difficult to recognize by standard methods. Gmira and Véron [9] proceeded by making the a priori substitution $v(t, x)=(\log t)^{n / 2} u(t, x)$ and estimating the new dependent variable $v(t, x)$. In the present paper, we proceed via a similar change of the solution $v(t, x)=e^{\varphi(t)} u(t, x)$, where the new unknown dependent variable $\varphi(t)$ is determined by the requirement of zero total mass of the nonlinearity (see Section 3). This condition enables us to consider a broader class of equations and to obtain a more detailed asymptotics.

In [12], we obtained the large-time asymptotic behavior of solutions to the Cauchy problem for the nonlinear Schrödinger equation with dissipation

$$
u_{t}+\mathscr{L} u+i|u|^{2} u=0, \quad x \in \mathbb{R}, t>0
$$

in the critical case, that is, when the symbol $L(\xi)$ of the linear pseudodifferential operator $\mathscr{L}$ has the following asymptotic representation $L(\xi) \sim \mu \xi^{2}$ in the origin $\xi \rightarrow 0$, where $\operatorname{Re} \mu>0$ and $\operatorname{Im} \mu \geq 0$.

In $[13,16]$, we considered the large-time asymptotics for solutions of the complex Landau-Ginzburg equation

$$
u_{t}-\mu \Delta u+a|u|^{q} u=0, \quad x \in \mathbb{R}^{n}, t>0
$$

in the critical case $q=2 / n$. The subcritical case $q \in(2 / n-\varepsilon, 2 / n)$, where $\varepsilon$ is sufficiently small and depends on the size of the data, was studied in [17], where $\mu, a \in \mathbf{C}, \operatorname{Re} \mu>0$, and $\mu$ and $a$ satisfy suitable angular conditions. In [14], the asymptotic expansion of small solutions to the Cauchy problem for the complex Landau-Ginzburg equation was considered.

Large-time asymptotics of equations with convective type nonlinearities in the critical case also was studied extensively (see [15] and the literature cited therein).

The aim of the present paper is to obtain the large-time asymptotic behavior of solutions to the Cauchy problem for the nonlinear evolution equations (1.1) in the critical case. Note that the method of $[7,9]$ does not work for (1.1). In the present paper, we generalize the approach developed in our previous papers [10, 12, 13, 14, 16, 17], where we considered mainly the case of Laplacian $\mathscr{L}=-\Delta$, the power nonlinearity $\mathcal{N}(u)=|u|^{q_{1}} u$, $0<q_{1} \leq 2 / n$ or nonlinearity of convective type $\mathcal{N}(u)=\partial_{x_{1}}|u|^{q_{2}+1}, 0<q_{2} \leq 1 / n$, and applied the $\mathbf{L}^{p}$-estimates of the Green operator $e^{t \Delta}$ to show the positivity of the value $\int \mathcal{N}\left(u_{1}\right) d x$, where $u_{1}$ is the first approximation of the solution and $\int\left(\mathcal{N}(u)-\mathcal{N}\left(u_{1}\right)\right) d x$ is the remainder term in our function space. In comparison with our previous works $[12,13,16]$, we are working here in the Lebesgue spaces for the Fourier transform of the solution in order to treat the case of nonlocal nonlinearities of nonconvective type involving derivatives of unknown function and to be able to show that $\int\left(\mathcal{N}(u)-\mathcal{N}\left(u_{1}\right)\right) d x$ is the remainder term in our function space defined below. To obtain the estimates of the remainder terms of the large-time asymptotic formulas, we have to assume that the initial data satisfy some decay condition at infinity. 
Suppose that the linear operator $\mathscr{L}$ satisfies the dissipation condition which in terms of the symbol $L(\xi)$ has the form

$$
\operatorname{Re} L(\xi) \geq \mu\{\xi\}^{\delta}\langle\xi\rangle^{v}
$$

for all $\xi \in \mathbb{R}$, where $\mu>0, v \geq 0$, and $\delta>0$. Also we suppose that the symbol is a smooth $L(\xi) \in \mathbf{C}^{1}(\mathbb{R})$ and has the estimate

$$
\left|\partial_{\xi}^{l} L(\xi)\right| \leq C\{\xi\}^{\delta-l}\langle\xi\rangle^{v}
$$

for all $\xi \in \mathbb{R} \backslash\{0\}, l=0,1$.

To find the asymptotic formulas for the solution, we assume that the symbol $L(\xi)$ has the following asymptotic representation in the origin:

$$
L(\xi)=L_{0}(\xi)+O\left(|\xi|^{\delta+\gamma}\right)
$$

for all $|\xi| \leq 1$, where $L_{0}(\xi)=\mu_{1}|\xi|^{\delta}+i \mu_{2}|\xi|^{\delta-1} \xi\left(\mu_{1}>0, \mu_{2} \in \mathbb{R}\right)$ and $\gamma \in(0,1)$.

We suppose that the symbols of the nonlinear operator $\mathcal{N}$ are such that

$$
\left|\partial_{\xi}^{l} a(t, \xi, y)\right| \leq C\{\xi-y\}^{-l}\left(\{\xi-y\}^{\alpha}\langle\xi-y\rangle^{\sigma}+\{y\}^{\alpha}\langle y\rangle^{\sigma}\right)
$$

for all $\xi, y \in \mathbb{R}, t>0$, and $l=0,1$, and

$$
\begin{aligned}
& \left|\partial_{\xi}^{l} b(t, \xi, y, z)\right| \\
& \quad \leq C\{\xi-y\}^{-l}\left(\{\xi-y\}^{\beta}\langle\xi-y\rangle^{\sigma}+\{y-z\}^{\beta}\langle y-z\rangle^{\sigma}+\{z\}^{\beta}\langle z\rangle^{\sigma}\right)
\end{aligned}
$$

for all $\xi, y, z \in \mathbb{R}, t>0$, and $l=0,1$, where $\alpha \geq 0, \beta \geq 0, \sigma=0$ if $v=0$, and $\sigma \in[0, v)$ if $\nu>0$. Here and below, we denote $\{\xi\}=|\xi| /\langle\xi\rangle,\langle\xi\rangle=\sqrt{1+\xi^{2}}$. We consider the case of nonlinearity of the nonconvective type, that is, we suppose that

$$
a(t, 0, y) \neq 0 \quad \text { or } \quad b(t, 0, y, z) \neq 0 .
$$

The critical case with respect to the large-time asymptotic behavior of solutions means that

$$
\delta=1+\alpha, \quad \delta=2+\beta
$$

We assume that the symbols of the nonlinearity have the asymptotics

$$
\begin{gathered}
a(t, 0, y)=a_{0}(y)+O\left(\{y\}^{\alpha+\gamma}\langle y\rangle^{\sigma}\right), \\
b(t, 0, y, z)=b_{0}(y, z)+O\left((\{y\}+\{z\})^{\beta+\gamma}(\langle y\rangle+\langle z\rangle)^{\sigma}\right)
\end{gathered}
$$

for all $y, z \in \mathbb{R}$ and $t>0$, where $\gamma \in(0,1), a_{0}(y)$ is homogeneous of order $\alpha$, and $b_{0}(y, z)$ is homogeneous of order $\beta$. For example, the equation

$$
\begin{gathered}
u_{t}+\left(\mu_{1}\left(-\partial_{x}^{2}\right)^{\delta / 2}+\mu_{2}\left(-\partial_{x}^{2}\right)^{(\delta-1) / 2} \partial_{x}+\mu_{3}\left(-\partial_{x}^{2}\right)^{v / 2}\right) u \\
+a_{1} \Pi_{j=1}^{2} \partial_{x}^{\alpha_{j}} u+b_{1} \Pi_{j=1}^{3} \partial_{x}^{\beta_{j}} u=0,
\end{gathered}
$$


where $\mu_{1}>0, \mu_{2}, a_{1}, b_{1} \in \mathbb{R}, \mu_{3} \geq 0,0<\delta<\nu, \alpha=\alpha_{1}+\alpha_{2}$, and $\beta=\beta_{1}+\beta_{2}+\beta_{3}$, satisfies conditions (1.16), (1.17), (1.18), (1.21), and (1.22). Also we suppose that the total mass of the initial data is not zero:

$$
\hat{u}_{0}(0)=\frac{1}{\sqrt{2 \pi}} \int_{\mathbb{R}} u_{0}(x) d x \neq 0 .
$$

Denote

$$
\begin{aligned}
\varkappa \equiv & \theta^{2} \int_{\mathbb{R}} a_{0}(y) e^{-L_{0}(-y)-L_{0}(y)} d y \\
& +\omega \theta^{3} \int_{\mathbb{R}^{2}} b_{0}(y, z) e^{-L_{0}(-y)-L_{0}(y-z)-L_{0}(z)} d y d z, \quad \theta=\hat{u}_{0}(0),
\end{aligned}
$$

where $\omega=0$ if $a_{0} \neq 0$ and $\omega=1$ if $a_{0} \equiv 0$. To obtain asymptotics of solutions in the critical case, we assume below that $x>0$. The condition $\varkappa>0$ implies the restriction on the nonlinearity and yields the positivity of the value $\int \mathcal{N}\left(u_{1}\right) d x>0$, where $u_{1}$ is the first approximation of the solution. We easily see that, for example, the equation

$$
u_{t}+\left(\mu_{1}\left(-\partial_{x}^{2}\right)^{\delta / 2}+\mu_{3}\left(-\partial_{x}^{2}\right)^{v / 2}\right) u+a_{1} \Pi_{j=1}^{2}\left(-\partial_{x}^{2}\right)^{\alpha_{j} / 2} u+b_{1} \Pi_{j=1}^{3}\left(-\partial_{x}^{2}\right)^{\beta_{j} / 2} u=0
$$

satisfies the condition $x>0$ if

$$
\begin{gathered}
\mu_{1}, \mu_{3}, a_{1}, b_{1}, \theta>0, \quad \delta<v, \\
\alpha_{1}+\alpha_{2}=\delta-1, \quad \beta_{1}+\beta_{2}+\beta_{3}=\delta-2 .
\end{gathered}
$$

Define $\mathbf{W}=\mathbf{A}^{0, \infty} \cap \mathbf{B}^{0,1} \cap \mathbf{D}^{0,0}$, where

$$
\begin{aligned}
\|\varphi\|_{\mathbf{A}^{0, \infty}} & =\|\hat{\varphi}(\xi)\|_{\mathbf{L}_{\xi}^{\infty}(|\xi| \leq 1)}, \\
\|\varphi\|_{\mathbf{B}^{0,1}} & =\|\hat{\varphi}(\xi)\|_{\mathbf{L}_{\xi}^{1}(|\xi| \geq 1)}, \\
\|\varphi\|_{\mathbf{D}^{0,0}} & =\left\|\left|\partial_{\xi}\right|^{\gamma} \hat{\varphi}(\xi)\right\|_{\mathbf{L}_{\xi}^{\infty}},
\end{aligned}
$$

and define the usual Lebesgue space $\mathbf{L}^{p}=\left\{\varphi \in \mathscr{S}^{\prime} ;\|\varphi\|_{\mathbf{L}^{p}}<\infty\right\}$, where

$$
\|\varphi\|_{\mathbf{L}^{p}}=\left(\int_{\mathbb{R}^{n}}|\varphi(x)|^{p} d x\right)^{1 / p}
$$

for $1 \leq p<\infty$ and $\|\varphi\|_{L^{\infty}}=\sup _{x \in \mathbb{R}^{n}}|\varphi(x)|$ for $p=\infty$, and we define the majorant of the fractional derivative of order $\gamma \in(0,1)$ as

$$
\left|\partial_{\xi}\right|^{\gamma} \phi(\xi) \equiv \int_{\mathbb{R}}|\phi(\xi-y)-\phi(\xi)||y|^{-1-\gamma} d y .
$$

Denote

$$
G_{0}(x)=\overline{\mathscr{F}} \xi \rightarrow x\left(e^{-L_{0}(\xi)}\right) .
$$

In the present paper we prove the following result. 
THEOREM 1.1. Assume that $u_{0} \in \mathrm{W}$ with sufficiently small norm

$$
\left\|u_{0}\right\|_{\mathbf{A}^{0, \infty}}+\left\|u_{0}\right\|_{\mathbf{B}^{0,1}}+\left\|u_{0}\right\|_{\mathbf{D}^{0,0}}=\varepsilon
$$

Suppose that $x>0$ and

$$
\theta=\widehat{u_{0}}(0)>0
$$

Then there exists a unique solution $u(t, x) \in \mathbf{L}^{\infty}((0, \infty) \times \mathbb{R}) \cap \mathbf{C}([0, \infty) ; \mathbf{W})$ of the Cauchy problem (1.1) satisfying the time-decay estimate

$$
\|u(t)\|_{\infty} \leq C\langle t\rangle^{-1 / \delta}(1+\varkappa \log \langle t\rangle)^{\omega / 2-1} .
$$

Furthermore, the asymptotic formula

$$
u(t, x)=\frac{\theta t^{-1 / \delta}}{1+\varkappa \log t} G_{0}\left(x t^{-1 / \delta}\right)+O\left(\frac{t^{-1 / \delta}}{(\log t) \log \log t}\right)
$$

is valid for $t \geq 1$ uniformly with respect to $x \in \mathbb{R}$ if $a_{0}(y) \neq 0$. In the case $a_{0}(y) \equiv 0$, the asymptotics

$$
u(t, x)=\frac{\theta t^{-1 / \delta}}{\sqrt{1+\varkappa \log t}} G_{0}\left(x t^{-1 / \delta}\right)+O\left(\frac{t^{-1 / \delta}}{\sqrt{\log t} \log \log t}\right)
$$

is true for $t \geq 1$ uniformly with respect to $x \in \mathbb{R}$.

REMARK 1.2. The conditions of the theorem on the initial data $u_{0}$ can also be expressed in terms of the usual weighted Sobolev spaces

$$
\mathbf{H}^{k, m}=\left\{\phi \in \mathbf{L}^{2}\|\phi\|_{\mathbf{H}^{k, m}} \equiv\left\|\langle x\rangle^{m}\left\langle i \partial_{x}\right\rangle^{k} \phi\right\|_{\mathbf{L}^{2}}<\infty\right\}
$$

as

$$
\left\|u_{0}\right\|_{\mathbf{H}^{\rho, 0}}+\left\|u_{0}\right\|_{\mathbf{H}^{0, \rho}} \leq \varepsilon, \quad \rho>\frac{1}{2}
$$

However, the conditions on the initial data $u_{0}$ are described more precisely in the norm W.

To help the reader to assimilate the assumptions regarding a pair of pseudodifferential operators and the initial data, we state the following list.

Assumptions on $\mathscr{L}\left(\langle\xi\rangle=\sqrt{1+\xi^{2}},\{\xi\}=|\xi| /\langle\xi\rangle\right)$. We assume the following:

(L1) $L(\xi) \in \mathbf{C}^{1}(\mathbb{R})$;

(L2) $\operatorname{Re} L(\xi) \geq \mu\{\xi\}^{\delta}\langle\xi\rangle^{v}, \mu>0, v \geq 0, \delta>0$;

(L3) $\left|\partial_{\xi}^{l} L(\xi)\right| \leq C\{\xi\}^{\delta-l}\langle\xi\rangle^{v}, l=0,1$;

(L4) (for detailed asymptotics) $L(\xi)=L_{0}(\xi)+O\left(|\xi|^{\delta+\gamma}\right.$ ) for all $|\xi| \leq 1$, where $L_{0}(\xi)=$ $\mu_{1}|\xi|^{\delta}+i \mu_{2}|\xi|^{\delta-1} \xi, \mu_{1}>0, \mu_{2} \in \mathbb{R}, \gamma \in(0,1)$. 
Assumptions on $\mathcal{N}$. We assume the following:

(N1) $a, b$ are continuous in $t>0$;

(N2) $|a(t, \xi, y)| \leq C\left(\langle\xi\rangle^{\kappa}+\langle y\rangle^{\kappa}\right)$, for some $\kappa>0$;

(N3) $|b(t, \xi, y, z)| \leq C\left(\langle\xi\rangle^{\kappa}+\langle y\rangle^{\kappa}+\langle z\rangle^{k}\right)$;

(N4) $\left|\partial_{\xi}^{l} a(t, \xi, y)\right| \leq C\{\xi-y\}^{-l}\left(\{\xi-y\}^{\alpha}\langle\xi-y\rangle^{\sigma}+\{y\}^{\alpha}\langle y\rangle^{\sigma}\right), l=0,1, \alpha \geq 0,0<$ $\sigma \leq v$

(N5) $\left|\partial_{\xi}^{l} b(t, \xi, y, z)\right| \leq C\{\xi-y\}^{-l}\left(\{\xi-y\}^{\beta}\langle\xi-y\rangle^{\sigma}+\{y-z\}^{\beta}\langle y-z\rangle^{\sigma}+\{z\}^{\beta}\langle z\rangle^{\sigma}\right)$, $l=0,1, \beta \geq 0$;

(N6) either $a(t, 0, y) \neq 0$ or $b(t, 0, y, z) \neq 0$;

(N7) $a(t, 0, y)=a_{0}(y)+O\left(\{y\}^{\alpha+\gamma}\langle y\rangle^{\sigma}\right), \gamma \in(0,1)$, and $a_{0}(y)$ is homogeneous of order $\alpha$

(N8) $b(t, 0, y, z)=b_{0}(y, z)+O\left((\{y\}+\{z\})^{\beta+\gamma}(\langle y\rangle+\langle z\rangle)^{\sigma}\right)$, and $b_{0}(y, z)$ is homogeneous of order $\beta$;

(N9) (critical case) $\delta=1+\alpha$ and $\delta=2+\beta$.

ASSUMPTIONS ON THE INITIAL DATA $u_{0}(x)$. We assume the following:

(D1) $\theta=(1 / \sqrt{2 \pi}) \int_{\mathbb{R}} u_{0}(x) d x \neq 0$;

(D2) the constant

$$
\begin{aligned}
\varkappa \equiv & \theta^{2} \int_{\mathbb{R}} a_{0}(y) e^{-L_{0}(-y)-L_{0}(y)} d y \\
& +\omega \theta^{3} \int_{\mathbb{R}^{2}} b_{0}(y, z) e^{-L_{0}(-y)-L_{0}(y-z)-L_{0}(z)} d y d z>0,
\end{aligned}
$$

where $\omega=0$ if $a_{0} \neq 0$ and $\omega=1$ if $a_{0} \equiv 0$;

(D3) $\left\|u_{0}\right\|_{\mathrm{H}^{\rho, 0}}+\left\|u_{0}\right\|_{\mathrm{H}^{0, \rho}} \leq \varepsilon$ for $\rho>1 / 2$, and some $\varepsilon>0$ sufficiently small.

REMARK 1.3. We now give examples of application of Theorem 1.1.

(1) In the case of cubic nonlinear heat equation (1.7) we have $a(t, \xi, y)=0, b(t, \xi, y, z)=$ $b_{0}(y, z)=1$, and $L(\xi)=L_{0}(\xi)=\xi^{2}$. The conditions (1.18) and (1.22) are fulfilled with $\sigma=\beta=0$ and $\delta=\nu=2$. Then for the small initial data $u_{0}$ such that $\theta>0$ and the norm $\left\|u_{0}\right\|_{\mathrm{H}^{\rho, 0}}+\left\|u_{0}\right\|_{\mathbf{H}^{0, \rho}} \leq \varepsilon, \rho>1 / 2$, the asymptotics

$$
u(t, x)=\frac{\theta}{\sqrt{4 \pi t} \sqrt{1+\chi \log t}} e^{-x^{2} / 2 t}+O\left(\frac{t^{-1 / 2}}{\sqrt{\log t} \log \log t}\right)
$$

is true for large $t$.

(2) As another example, we consider the cubic nonlinear heat equation with convection

$$
u_{t}+u u_{x}+u^{3}-u_{x x}=0, \quad x \in \mathbb{R}, t>0 .
$$

It represents an interaction of the quadratic convective nonlinearity with cubic nonconvective nonlinear term; both are critical. We have $a(t, \xi, y)=\xi, a_{0}(y)=0, b(t, \xi, y, z)=$ $b_{0}(y, z)=1$, and $L(\xi)=L_{0}(\xi)=\xi^{2}$. The conditions (1.18) and (1.22) are fulfilled with $\sigma=\alpha=1, \beta=0$, and $\delta=\nu=2$. Then for small initial data $u_{0}$ such that $\theta>0$ and the norm $\left\|u_{0}\right\|_{\mathbf{H}^{\rho, 0}}+\left\|u_{0}\right\|_{\mathbf{H}^{0, \rho}} \leq \varepsilon, \rho>1 / 2$, the asymptotics (1.40) is true for large $t$. Thus the cubic nonconvective nonlinearity dominates under convective term and defines the main term of the large-time asymptotic behavior of solutions. 
(3) For the potential Whitham equation (1.8), we have $a(t, \xi, y)=-(\xi-y) y, b(t, \xi, y, z)$ $=0, L(\xi)=|\xi|^{3}-i \xi^{3}, a_{0}(y)=y^{2}$, and $L_{0}(\xi)=|\xi|^{3}-i \xi^{3}$. The conditions (1.17) and (1.21) are fulfilled with $\sigma=\alpha=2$ and $\delta=v=3$. Then for small initial data $u_{0}$ such that $\theta>0$ and the norm $\left\|u_{0}\right\|_{\mathbf{H}^{\rho, 0}}+\left\|u_{0}\right\|_{\mathbf{H}^{0, \rho}} \leq \varepsilon, \rho>1 / 2$, the asymptotics

$$
u(t, x)=\frac{\theta t^{-1 / 3}}{1+\chi \log t} G_{0}\left(x t^{-1 / 3}\right)+O\left(\frac{t^{-1 / 3}}{(\log t) \log \log t}\right)
$$

is valid. Note that there is no blowup for the Whitham equation (1.8), that is, all solutions exist globally in time (see [20]) even if $\theta<0$. It is interesting to know the character of the large-time asymptotic behavior of solutions in the case $\theta<0$. As far as we know, this is an open problem.

We organize the rest of the paper as follows. Some preliminary estimates of the Green operator solving the linearized Cauchy problem corresponding to (1.1) are obtained in Section 2. The proof of Theorem 1.1 will be given in Section 3.

2. Preliminary lemmas. The solution of the linear Cauchy problem

$$
\begin{gathered}
u_{t}+\mathscr{L} u=f(t, x), \quad x \in \mathbb{R}, t>0, \\
u(0, x)=u_{0}(x), \quad x \in \mathbb{R}
\end{gathered}
$$

can be written by the Duhamel formula

$$
u(t)=\mathscr{G}(t) u_{0}+\int_{0}^{t} \mathscr{G}(t-\tau) f(\tau) d \tau,
$$

where the Green operator $\mathscr{G}$ is given by

$$
\mathscr{G}(t) \phi=\overline{\mathscr{F}}_{\xi \rightarrow x}\left(e^{-L(\xi) t} \hat{\phi}(\xi)\right) .
$$

We first collect some preliminary estimates for the Green operator $\mathscr{G}(t)$ in the norms

$$
\begin{aligned}
\|\varphi(t)\|_{\mathbf{A}^{\rho, p}} & =\left\||\xi|^{\rho} \hat{\varphi}(t, \xi)\right\|_{\mathbf{L}_{\xi}^{p}(|\xi| \leq 1)}, \\
\|\varphi(t)\|_{\mathbf{B}^{s, p}} & =\left\||\xi|^{s} \hat{\varphi}(t, \xi)\right\|_{\mathbf{L}_{\xi}^{p}(|\xi| \geq 1)}, \\
\|\varphi(t)\|_{\mathbf{D}^{\rho, s}} & =\left\|\left|\partial_{\xi}\right|^{\gamma}\{\xi\}^{\rho}\langle\xi\rangle^{s} \hat{\varphi}(t, \xi)\right\|_{\mathbf{L}_{\xi}^{\infty},}
\end{aligned}
$$

where $\rho, s \in \mathbb{R}$ and $\gamma \in(0,1)$. The norm $\mathrm{A}^{\rho, p}$ is responsible for the large-time asymptotic properties of solutions and the norm $\mathbf{B}^{s, p}$ describes the regularity of solutions.

LEMMA 2.1. Let the linear operator $\mathscr{L}$ satisfy the dissipation conditions (1.14) and (1.15) and $\hat{\varphi}(0)=0$. Then the estimates are valid for all $t>0$ :

$$
\|\mathscr{G}(t) \varphi\|_{\mathrm{A}^{\rho, p}} \leq C\langle t\rangle^{-(1 / \delta)(\rho+1 / p-1 / q)}\|\varphi\|_{\mathrm{A}^{0, q}}
$$

for $\rho \geq 0$ if $p=q$ and $\rho+1 / p-1 / q>0$ if $1 \leq p<q \leq \infty$;

$$
\|\mathscr{G}(t) \varphi\|_{\mathbf{A}^{\rho, p}} \leq C\langle t\rangle^{-(1 / \delta)(\rho+\gamma+1 / p)}\|\varphi\|_{\mathbf{D}^{0,0}}
$$


for $\rho+\gamma \geq 0$ if $p=\infty$ and for $\rho+\gamma+1 / p>0$ if $1 \leq p<\infty$;

$$
\|\mathscr{G}(t) \varphi\|_{\mathbf{B}^{s, p}} \leq C e^{-(\mu / 2) t}\{t\}^{-s / v}\|\varphi\|_{\mathbf{B}^{0, p}},
$$

where $1 \leq p \leq \infty, s \geq 0$, and in the case $v=0, s=0$; and

$$
\begin{aligned}
\|\mathscr{G}(t) \varphi\|_{\mathbf{D}^{\rho, s}} \leq & C\langle t\rangle^{-\rho / \delta}\{t\}^{-s / v}\left(\|\varphi\|_{\mathbf{D}^{0,0}}+\|\varphi\|_{\mathbf{A}^{0, \infty}}+\|\varphi\|_{\mathbf{B}^{0, \infty}}\right) \\
& +C\langle t\rangle^{-\rho / \delta+\gamma / 2 \delta}\{t\}^{-s / v}\left(\|\varphi\|_{\mathbf{A}^{0, \infty}}+\|\varphi\|_{\mathbf{B}^{0, \infty}}\right)^{1 / 2}\|\varphi\|_{\mathbf{D}^{0,0}}^{1 / 2},
\end{aligned}
$$

where $1 \leq p \leq q \leq \infty, s \geq 0, \rho \geq 0$, and $\gamma \in[0,1)$ is such that $\gamma<\delta$ if $\rho=0$ and $\gamma<\min (\rho, \delta)$ if $\rho>0$. In the case $v=0, s=0$.

Proof. By virtue of dissipation conditions (1.14), we have

$$
|\xi|^{\rho}\left|e^{-t L(\xi)}\right| \leq C|\xi|^{\rho} e^{-t \mu|\xi|^{\delta}} \leq C\langle t\rangle^{-\rho / \delta} e^{-t(\mu / 2)|\xi|^{\delta}}
$$

for all $t>0$ and $|\xi| \leq 1$, where $\rho \geq 0$. By (2.9), changing the variable $\eta=\xi\langle t\rangle^{1 / \delta}$, we get in the case $\rho+1 / r>0,1 \leq r<\infty$,

$$
\begin{aligned}
\left\||\xi|^{\rho} e^{-t L(\xi)}\right\|_{L_{\xi}^{r}(|\xi| \leq 1)} & =\left(\int_{|\xi| \leq 1} d \xi|\xi|^{r \rho} e^{-t r L(\xi)}\right)^{1 / r} \\
& \leq C\langle t\rangle^{-(1 / \delta)(\rho+1 / r)}\left(\int_{|\eta| \leq\langle t\rangle} d \eta \delta|\eta|^{r \rho} e^{-\mu|\eta|^{\delta}}\right)^{1 / r} \\
& \leq C\langle t\rangle^{-(1 / \delta)(\rho+1 / r)}
\end{aligned}
$$

and for $\rho \geq 0$,

$$
\left\||\xi|^{\rho} e^{-t L(\xi)}\right\|_{L_{\xi}^{\infty}(|\xi| \leq 1)}=C\langle t\rangle^{-\rho / \delta}\left\|e^{-t \mu / 2|\xi|^{\delta}}\right\|_{L_{\xi}^{\infty}(|\xi| \leq 1)} \leq C\langle t\rangle^{-\rho / \delta}
$$

whence

$$
\begin{aligned}
\|\mathscr{G}(t) \varphi\|_{\mathrm{A}^{\rho, p}} & =\left\||\xi|^{\rho} e^{-t L(\xi)} \hat{\varphi}(\xi)\right\|_{\mathbf{L}_{\xi}^{p}(|\xi| \leq 1)} \\
& \leq C\left\||\xi|^{\rho} e^{-t L(\xi)}\right\|_{\mathbf{L}_{\xi}^{p q /(q-p)}(|\xi| \leq 1)}\|\hat{\varphi}(\xi)\|_{\mathbf{L}_{\xi}^{q}(|\xi| \leq 1)} \\
& \leq C\langle t\rangle^{-(1 / \delta)(\rho+1 / p-1 / q)}\|\varphi\|_{\mathbf{A}^{0, q}}
\end{aligned}
$$

for $\rho+1 / p-1 / q>0$ if $1 \leq p<q \leq \infty$ and for $\rho \geq 0$ if $p=q$.

Note that $\phi \in \mathbf{C}(\mathbb{R})$ if $\left\|\left|\partial_{\xi}\right|^{\gamma} \phi(\xi)\right\|_{\mathbf{L}_{\xi}^{\infty}<\infty}$. Then applying the identity

$$
1=\frac{\gamma}{2^{\gamma}-1}|\xi|^{\gamma} \int_{|\xi| / 2}^{|\xi|} \eta^{-1-\gamma} d \eta
$$


via the mean value theorem, we see that for every $\xi>0$, there exists $\zeta(\xi) \in[0, \xi / 2]$ such that

$$
\begin{aligned}
|\phi(\zeta(\xi))-\phi(\xi)| & =\frac{\gamma}{2^{\gamma}-1} \xi^{\gamma} \int_{\xi / 2}^{\xi}|\phi(\xi-\eta)-\phi(\xi)| \eta^{-1-\gamma} d \eta \\
& \leq C \xi^{\gamma} \int_{\mathbb{R}}|\phi(\xi-\eta)-\phi(\xi)||\eta|^{-1-\gamma} d \eta \\
& \leq\left.\left. C|\xi|^{\gamma}|| \partial_{\xi}\right|^{\gamma} \phi(\xi)\right|_{L_{\xi}^{\infty} .}
\end{aligned}
$$

We denote $\xi_{0}=\xi$ and define a sequence $\xi_{n}=\zeta\left(\xi_{n-1}\right) \in\left[0, \xi 2^{-n}\right]$ for $n \geq 1$. Since

$$
\left|\phi\left(\xi_{n}\right)-\phi\left(\xi_{n-1}\right)\right| \leq C 2^{-n \gamma}|\xi|^{\gamma} \sup _{\xi \in \mathbb{R}}|\partial \xi|^{\gamma} \phi(\xi)
$$

we get

$$
\begin{aligned}
|\phi(0)-\phi(\xi)| & =\lim _{n \rightarrow \infty}\left|\phi\left(\xi_{n}\right)-\phi(\xi)\right| \\
& \leq \sum_{n=1}^{\infty}\left|\phi\left(\xi_{n}\right)-\phi\left(\xi_{n-1}\right)\right| \\
& \leq\left. C|\xi|^{\gamma} \sum_{n=1}^{\infty} 2^{-n \gamma}||\left|\partial_{\xi}\right|^{\gamma} \phi(\xi)\right|_{L_{\xi}^{\infty}} \\
& \leq\left. C|\xi|^{\gamma}||\left|\partial_{\xi}\right|^{\gamma} \phi(\xi)\right|_{\mathbf{L}_{\xi}^{\infty}} .
\end{aligned}
$$

The case $\xi<0$ is considered in the same manner. Thus, for all $\xi \in \mathbb{R}$,

$$
|\xi|^{-\gamma}|\hat{\varphi}(\xi)-\hat{\varphi}(0)| \leq C||\left|\partial_{\xi}\right|^{\gamma} \hat{\varphi}(\xi)\left\|_{\mathbf{L}_{\xi}^{\infty}} \leq C\right\| \varphi \|_{\mathbf{D}^{0,0}}
$$

Therefore, by (2.10), (2.11), and (2.17), via condition $\hat{\varphi}(0)=0$, we get

$$
\begin{aligned}
\|\mathscr{G}(t) \varphi\|_{\mathbf{A}^{\rho, p}} & =\left\||\xi|^{\rho} e^{-t L(\xi)} \hat{\varphi}(\xi)\right\|_{\mathbf{L}_{\xi}^{p}(|\xi| \leq 1)} \\
& \leq C\left\||\xi|^{\rho+\gamma} e^{-t L(\xi)}\right\|_{\mathbf{L}_{\xi}^{p}(|\xi| \leq 1)}\left\||\xi|^{-\gamma}(\hat{\varphi}(\xi)-\hat{\varphi}(0))\right\|_{\mathbf{L}_{\xi}^{\infty}(|\xi| \leq 1)} \\
& \leq C\langle t\rangle^{-(1 / \delta)(\rho+\gamma+1 / p)}\|\varphi\|_{\mathbf{D}^{0,0}}
\end{aligned}
$$

for $\rho+\gamma+1 / p>0$ if $1 \leq p<\infty$ and for $\rho+\gamma \geq 0$ if $p=\infty$. Thus, the second estimate of the lemma is true. For the third estimate, we have by condition (1.14)

$$
|\xi|^{s}\left|e^{-t L(\xi)}\right| \leq C|\xi|^{s} e^{-t \mu|\xi|^{v}} \leq C e^{-(\mu / 2) t}\{t\}^{-s / v}
$$

for all $t>0$ and $|\xi| \geq 1$, where $s \geq 0$. Therefore,

$$
\begin{aligned}
\|\mathscr{G}(t) \varphi\|_{\mathbf{B}^{s, p}} & =\left\||\xi|^{s} e^{-t L(\xi)} \hat{\varphi}(\xi)\right\|_{\mathbf{L}_{\xi}^{p}(|\xi| \geq 1)} \\
& \leq C\left\|\left.|| \xi\right|^{s} e^{-t L(\xi)}\right\|_{\mathbf{L}_{\xi}^{\infty}(|\xi| \geq 1)}\|\hat{\varphi}(\xi)\|_{\mathbf{L}_{\xi}^{p}(|\xi| \geq 1)} \\
& \leq C e^{-(\mu / 2) t}\{t\}^{-s / v}\|\varphi\|_{\mathbf{B}^{0, p}} .
\end{aligned}
$$


We now prove the fourth estimate. By the Leibnitz rule, we have

$$
\begin{aligned}
|\partial \xi|^{\gamma}\left(\{\xi\}^{\rho}\langle\xi\rangle^{s} e^{-t L(\xi)} \hat{\varphi}(\xi)\right) \leq & \{\xi\}^{\rho}\langle\xi\rangle^{s} e^{-L(\xi) t}|\partial \xi|^{\gamma} \hat{\varphi}(\xi) \\
& +\int_{\mathbb{R}} K(t, \xi, \eta)|\hat{\varphi}(\xi-\eta)||\eta|^{-1-\gamma} d \eta,
\end{aligned}
$$

where

$$
K(t, \xi, \eta) \equiv\left|\{\xi-\eta\}^{\rho}\langle\xi-\eta\rangle^{s} e^{-L(\xi-\eta) t}-\{\xi\}^{\rho}\langle\xi\rangle^{s} e^{-L(\xi) t}\right|
$$

Via the first and the third estimates of the lemma, we have

$$
\begin{aligned}
\left\|\{\xi\}^{\rho}\langle\xi\rangle^{s} e^{-L(\xi) t}\left|\partial_{\xi}\right|^{\gamma} \hat{\varphi}(\xi)\right\|_{L_{\xi}^{\infty}} \leq & C\langle t\rangle^{-\rho / \delta}\left\|\left|\partial_{\xi}\right|^{\gamma} \hat{\varphi}(\xi)\right\|_{L_{\xi}^{\infty}(|\xi| \leq 1)} \\
& +C e^{-(\mu / 2) t}\{t\}^{-s / v}\left\|\left|\partial_{\xi}\right|^{\gamma} \hat{\varphi}(\xi)\right\|_{L_{\xi}^{\infty}(|\xi| \geq 1)} \\
\leq & C\langle t\rangle^{-\rho / \delta}\{t\}^{-s / v}\|\varphi\|_{\mathbf{D}^{0,0}}
\end{aligned}
$$

therefore,

$$
\begin{aligned}
\|\mathscr{G}(t) \varphi\|_{\mathbf{D}^{\rho, s}} \leq & C\langle t\rangle^{-\rho / \delta}\{t\}^{-s / v}\|\varphi\|_{\mathbf{D}^{0,0}} \\
& +C\left\|\int_{|\eta|>\langle t\rangle^{-1 / \delta}}|\hat{\varphi}(\xi-\eta)| K(t, \xi, \eta)|\eta|^{-1-\gamma} d \eta\right\|_{\mathbf{L}_{\xi}^{\infty}} \\
& +C\left\|\int_{|\eta| \leq \min \left(|\xi| / 2,\langle t\rangle^{-1 / \delta}\right)}|\hat{\varphi}(\xi-\eta)| K(t, \xi, \eta)|\eta|^{-1-\gamma} d \eta\right\|_{\mathbf{L}_{\xi}^{\infty}} \\
& +C\left\|\int_{|\xi| / 2 \leq|\eta| \leq\langle t\rangle^{-1 / \delta}}|\hat{\varphi}(\xi-\eta)| K(t, \xi, \eta)|\eta|^{-1-\gamma} d \eta\right\|_{\mathbf{L}_{\xi}^{\infty}}
\end{aligned}
$$

We estimate the second summand in (2.24) in view of condition (1.14) and estimate (2.17):

$$
\begin{aligned}
& \left\|\int_{|\eta|>\langle t\rangle^{-1 / \delta}}|\hat{\varphi}(\xi-\eta)| K(t, \xi, \eta)|\eta|^{-1-\gamma} d \eta\right\|_{\mathbf{L}_{\xi}^{\infty}} \\
& \leq\|\hat{\varphi}(\xi-\eta)\|_{\mathbf{L}_{\xi}^{\infty}}^{1 / 2}\|\varphi\|_{\mathbf{D}^{0,0}}^{1 / 2}\left\|\int_{|\eta|>\langle t\rangle^{-1 / \delta}}|\xi-\eta|^{\gamma / 2} K(t, \xi, \eta)|\eta|^{-1-\gamma} d \eta\right\|_{\mathbf{L}_{\xi}^{\infty}} \\
& \leq C\left\|\{\xi\}^{\rho+\gamma / 2}\langle\xi\rangle^{s} e^{-t \operatorname{Re} L(\xi)}\right\|_{\mathbf{L}_{\xi}^{\infty}}\|\hat{\varphi}(\xi)\|_{\mathbf{L}_{\xi}^{\infty}}^{1 / 2}\|\varphi\|_{\mathbf{D}^{0,0}}^{1 / 2} \int_{|\eta|>\langle t\rangle^{-1 / \delta}} \frac{d \eta}{|\eta|^{1+\gamma}} \\
& \quad+C\left\|\{\xi\}^{\rho}\langle\xi\rangle^{s} e^{-t \operatorname{Re} L(\xi)}\right\|_{\mathbf{L}_{\xi}^{\infty}}\|\hat{\varphi}(\xi)\|_{\mathbf{L}_{\xi}^{\infty}}^{1 / 2}\|\varphi\|_{\mathbf{D}^{0,0}}^{1 / 2} \int_{|\eta|>\langle t\rangle^{-1 / \delta}} \frac{d \eta}{|\eta|^{1+\gamma / 2}} \\
& \leq C\langle t\rangle^{-\rho / \delta+\gamma / 2 \delta}\{t\}^{-s / v}\left(\|\varphi\|_{\mathbf{A}^{0, \infty}}+\|\varphi\|_{\mathbf{B}^{0, \infty}}\right)^{1 / 2}\|\varphi\|_{\mathbf{D}^{0,0}}^{1 / 2} .
\end{aligned}
$$


In the case $|\eta| \leq \min \left(|\xi| / 2,\langle t\rangle^{-1 / \delta}\right)$, we get by condition (1.15)

$$
\begin{aligned}
K(t, \xi, \eta) & =\left|\int_{0}^{1} \partial_{h}\left(\{\xi-h \eta\}^{\rho}\langle\xi-h \eta\rangle^{s} e^{-t L(\xi-h \eta)}\right) d h\right| \\
& \leq C|\eta|\left\|\left(t\{\zeta\}^{\delta-1}\langle\zeta\rangle^{v}+\rho\{\zeta\}^{-1}\right)\{\zeta\}^{\rho}\langle\zeta\rangle^{s} e^{-\mu t\{\zeta\}^{\delta}\langle\zeta\rangle^{v}}\right\|_{\mathbf{L}_{\zeta}^{\infty}(|\zeta| \geq|\xi| / 2)} \\
& \leq C|\eta|\{\xi\}^{-1}\left\|\left(1+t\{\zeta\}^{\delta}\langle\zeta\rangle^{v}\right)\{\zeta\}^{\rho}\langle\zeta\rangle^{s} e^{-C t\{\zeta\}^{\delta}\langle\zeta\rangle^{v}}\right\|_{\mathbf{L}_{\zeta}^{\infty}} \\
& \leq C|\eta|\{\xi\}^{-1}\{t\}^{-s / v}\langle t\rangle^{-\rho / \delta},
\end{aligned}
$$

therefore,

$$
\begin{aligned}
C\left\|\int_{|\eta| \leq \min \left(|\xi| / 2,\langle t\rangle^{-1 / \delta}\right)}|\hat{\varphi}(\xi-\eta)| K(t, \xi, \eta)|\eta|^{-1-\gamma} d \eta\right\|_{\mathbf{L}_{\xi}^{\infty}} \\
\leq C\{t\}^{-s / v}\langle t\rangle^{-\rho / \delta}\left(\|\varphi\|_{\mathbf{D}^{0,0}}+\|\hat{\varphi}(\xi)\|_{\mathbf{L}_{\xi}^{\infty}}\right) \\
\quad \times\left\|\{\xi\}^{\gamma-1} \int_{|\eta| \leq \min \left(|\xi| / 2,\langle t\rangle^{-1 / \delta}\right)}|\eta|^{-\gamma} d \eta\right\|_{\mathbf{L}_{\xi}^{\infty}} \\
\quad \leq C\{t\}^{-s / v}\langle t\rangle^{-\rho / \delta}\left(\|\varphi\|_{\mathbf{D}^{0,0}}+\|\varphi\|_{\mathbf{A}^{0, \infty}}+\|\varphi\|_{\mathbf{B}^{0, \infty}}\right) .
\end{aligned}
$$

And in the case $|\xi| / 2 \leq|\eta| \leq\langle t\rangle^{-1 / \delta}$, via estimate

$$
\begin{aligned}
K(t, \xi, \eta) & \leq C\left|\int_{\xi}^{\xi-\eta} e^{-\operatorname{Re} L(y) t}\left(t\left|L^{\prime}(y)\right|+\rho\{y\}^{-1}\right)\{y\}^{\rho} d y\right| \\
& \leq C \int_{\xi}^{\xi-\eta}\left(t\{y\}^{\delta+\rho-1}+\rho\{y\}^{\rho-1}\right) d y \\
& \leq C|\eta|^{\rho}\left(t|\eta|^{\delta}+\rho\right),
\end{aligned}
$$

we obtain

$$
\begin{aligned}
\left\|\int_{|\xi| / 2 \leq|\eta| \leq\langle t\rangle^{-1 / \delta}}|\hat{\varphi}(\xi-\eta)| K(t, \xi, \eta)|\eta|^{-1-\gamma} d \eta\right\|_{\mathbf{L}_{\xi}^{\infty}} \\
\leq C\|\varphi\|_{\mathbf{D}^{0,0}} \int_{|\eta| \leq\langle t\rangle^{-1 / \delta}}\left(t|\eta|^{\delta}+\rho\right)|\eta|^{\rho-1} d \eta \\
\leq C\langle t\rangle^{-\rho / \delta}\|\varphi\|_{\mathbf{D}^{0,0}} .
\end{aligned}
$$

Substitution of (2.25), (2.27), and (2.29) into (2.24) yields the fourth estimate of the lemma. Lemma 2.1 is proved.

Define $\varphi_{0}(t)$ by

$$
\mathscr{G}_{0}(t) \phi=\overline{\mathscr{F}}_{\xi \rightarrow x}\left(e^{-L_{0}(\xi) t} \hat{\phi}(\xi)\right),
$$


where the symbol $L_{0}(\xi)=\mu_{1}|\xi|^{\delta}+i \mu_{2}|\xi|^{\delta-1} \xi$ is homogeneous, $\mu_{1}, \mu_{2} \in \mathbb{R}$, and

$$
G_{0}(x)=\overline{\mathscr{F}} \xi \rightarrow x\left(e^{-L_{0}(\xi)}\right) .
$$

In the next lemma, we estimate a difference $\mathscr{G}(t)-\mathscr{G}_{0}(t)$.

LEMMA 2.2. Let the linear operator $\mathscr{L}$ satisfy conditions (1.14) and (1.16), then the estimates

$$
\begin{gathered}
\left\|\left(\mathscr{G}(t)-\mathscr{G}_{0}(t)\right) \phi\right\|_{\mathrm{A}^{\rho, p}} \leq C\langle t\rangle^{-(\rho+\gamma) / \delta-1 / \delta p}\|\phi\|_{\mathrm{A}^{0, \infty}}, \\
\left\|\mathscr{G}_{0}(t) \phi-t^{-1 / \delta} \hat{\phi}(0) G_{0}\left(t^{-1 / \delta}(\cdot)\right)\right\|_{\mathrm{A}^{\rho, p}} \leq C\langle t\rangle^{-(\rho+\gamma) / \delta-1 / \delta p}\|\phi\|_{\mathbf{D}^{0,0}}
\end{gathered}
$$

are valid for all $t>0$, where $1 \leq p \leq \infty, \rho \geq 0$, and $\gamma \in[0,1)$ is such that $\gamma<\delta$ if $\rho=0$ and $\gamma<\min (\rho, \delta)$ if $\rho>0$.

Proof. By virtue of the dissipation condition (1.14) and asymptotics (1.16), we have

$$
\left|e^{-t L(\xi)}-e^{-t L_{0}(\xi)}\right|=\left|\int_{t L(\xi)}^{t L_{0}(\xi)} e^{-\eta} d \eta\right| \leq C t\left|L(\xi)-L_{0}(\xi)\right| e^{-C t|\xi|^{\delta}} \leq C t|\xi|^{\delta+\gamma} e^{-C t|\xi|^{\delta}}
$$

for all $t>0$ and $|\xi| \leq 1$, whence

$$
\begin{aligned}
\left\|\left(\mathscr{G}(t)-\mathscr{G}_{0}(t)\right) \phi\right\|_{\mathrm{A}^{\rho, p}} & =\left\||\xi|^{\rho}\left(e^{-t L(\xi)}-e^{-t L_{0}(\xi)}\right) \hat{\phi}(\xi)\right\|_{\mathbf{L}_{\xi}^{p}(|\xi| \leq 1)} \\
& \leq C t\left\||\xi|^{\delta+\rho+\gamma} e^{-C t|\xi|^{\delta}}\right\|_{\mathbf{L}_{\xi}^{p}(|\xi| \leq 1)}\|\hat{\phi}(\xi)\|_{\mathbf{L}_{\xi}^{\infty}(|\xi| \leq 1)} \\
& \leq C\langle t\rangle^{-(\rho+\gamma) / \delta-1 / \delta p}\|\phi\|_{\mathbf{A}^{0, \infty}} .
\end{aligned}
$$

Thus, the first estimate is true.

We prove the second estimate by (2.9) and (2.17); we get

$$
\begin{aligned}
\| \mathscr{G}_{0}( & t) \phi-t^{-1 / \delta} \hat{\phi}(0) G_{0}\left(t^{-1 / \delta}(\cdot)\right) \|_{\mathrm{A}^{\rho, p}} \\
= & \left\||\xi|^{\rho} e^{-t L_{0}(\xi)}(\hat{\phi}(\xi)-\hat{\phi}(0))\right\|_{\mathbf{L}_{\xi}^{p}(|\xi| \leq 1)} \\
\leq & C\left\|\left.|| \xi\right|^{\rho+\gamma} e^{-t L_{0}(\xi)}\right\|_{\mathbf{L}_{\xi}^{p}(|\xi| \leq 1)}\left\||\xi|^{-\gamma}(\hat{\phi}(\xi)-\hat{\phi}(0))\right\|_{\mathbf{L}_{\xi}^{\infty}(|\xi| \leq 1)} \\
\leq & C\langle t\rangle^{-(\rho+\gamma) / \delta-1 / \delta p}\|\phi\|_{\mathbf{D}^{0,0}} .
\end{aligned}
$$

Therefore, the second estimate of the lemma is valid. Hence, lemma 2.2 is proved.

In the next lemma, we estimate the Green operator $\mathscr{G}(t)$ in our basic norms $\|\cdot\|_{\mathrm{X}}$ and $\|\cdot\|_{\mathrm{Y}}$ which depend on the order of $L(\xi)$ (see conditions (1.14), (1.15), and (1.16)) and on the symbols $a$ and $b$ (see (1.17), (1.18), (1.21), and (1.22)), namely, they depend on 
$\delta, \nu, \alpha, \beta, \sigma$, and $\gamma$ which are defined as follows:

$$
\begin{aligned}
\|\phi\|_{\mathbf{X}}= & \sup _{\rho \in[-\gamma, \alpha+\gamma]} \sup _{t>0}\langle t\rangle^{(\rho+1) / \delta}\|\phi(t)\|_{\mathbf{A}^{\rho, 1}} \\
& +\sup _{\rho \in[0, \alpha+\gamma]} \sup _{t>0}\langle t\rangle^{\rho / \delta}\|\phi(t)\|_{\mathbf{A}^{\rho, \infty}} \\
& +\sup _{s \in[0, \sigma]} \sup _{1 \leq p \leq \infty} \sup _{t>0}\{t\}^{s / v}\langle t\rangle^{1+\gamma / \delta+1 / \delta p}\|\phi(t)\|_{\mathbf{B}^{s, p}} \\
& +\sup _{\rho=0, \alpha, \beta} \sup _{s \in[0, \sigma]} \sup _{t>0}\{t\}^{s / v}\langle t\rangle^{(\rho-\gamma) / \delta}\|\phi(t)\|_{\mathbf{D}^{\rho, s}}, \\
\|\phi\|_{\mathbf{Y}}= & \sup _{1 \leq p \leq \infty} \sup _{t>0}\langle t\rangle^{1+1 / \delta p}\|\phi(t)\|_{\mathbf{A}^{0, p}} \\
& +\sup _{1 \leq p \leq \infty} \sup _{t>0}\langle t\rangle^{1+\gamma / \delta+1 / \delta p}\{t\}^{\sigma / v}\|\phi(t)\|_{\mathbf{B}^{0, p}} \\
& +\sup _{t>0}\langle t\rangle^{1-\gamma / \delta}\{t\}^{\sigma / v}\|\phi(t)\|_{\mathbf{D}^{0,0}},
\end{aligned}
$$

where $\gamma \in(0, \min (1, \delta))$ is such that $\gamma<\alpha$ if $\alpha>0$ and $\gamma<\beta$ if $\beta>0$. Define the function $g(t)$ :

$$
g(t)=1+\kappa \log \langle t\rangle
$$

with some $\kappa>0$.

LEMMA 2.3. Let the function $f(t, x)$ have a zero mean value $\hat{f}(t, 0)=0$. Then the inequality

$$
\left\|g(t) \int_{0}^{t} g^{-1}(\tau) \mathscr{G}(t-\tau) f(\tau) d \tau\right\|_{\mathbf{X}} \leq C\|f\|_{\mathrm{Y}}
$$

is valid, provided that the right-hand side is finite.

Proof. Via the condition of the lemma for the function $g(t)$, we have the estimate $\langle t\rangle^{-\gamma / 4 \delta} \leq C g^{-1}(t)$ and since the $\operatorname{limit}_{t \rightarrow \infty}(g(t) / g(\sqrt{t}))$ exists, so

$$
\sup _{\tau \in[\sqrt{t}, t]} g^{-1}(\tau) \leq C g^{-1}(t)
$$

Hence, by virtue of the first two estimates of Lemma 2.1 and (2.39), we obtain for $\rho \in$ $[-\gamma, \alpha+\gamma]$ if $p=1$ and $\rho \in[0, \alpha+\gamma]$ if $p=\infty$

$$
\begin{aligned}
& \left\|\int_{0}^{t} g^{-1}(\tau) \mathscr{G}(t-\tau) f(\tau) d \tau\right\|_{\mathrm{A}^{\rho, p}} \\
& \leq C \int_{0}^{\sqrt{t}}\langle t-\tau\rangle^{-(\rho+\gamma) / \delta-1 / \delta p}\langle\tau\rangle^{\gamma / \delta-1}\{\tau\}^{-s / v} d \tau \\
& \quad \times \sup _{\tau>0}\langle\tau\rangle^{1-\gamma / \delta}\{\tau\}^{s / v}\|f(\tau)\|_{\mathbf{D}^{0,0}}
\end{aligned}
$$




$$
\begin{aligned}
& +C g^{-1}(t) \int_{\sqrt{t}}^{t / 2}\langle t-\tau\rangle^{-(\rho+\gamma) / \delta-1 / \delta p}\langle\tau\rangle^{\gamma / \delta-1}\{\tau\}^{-s / v} d \tau \\
& \times \sup _{\tau>0}\langle\tau\rangle^{1-\gamma / \delta}\{\tau\}^{s / v}\|f(\tau)\|_{\mathbf{D}^{0,0}} \\
& +C g^{-1}(t) \int_{t / 2}^{t}\langle t-\tau\rangle^{-\rho / \delta}\langle\tau\rangle^{-1 / \delta p-1} d \tau \\
& \times \sup _{\tau>0}\langle\tau\rangle^{1+1 / \delta p}\|f(\tau)\|_{\mathbf{A}^{0, p}} \\
& \leq C\langle t\rangle^{-\rho / \delta-1 / \delta p}\left(\langle t\rangle^{-\gamma / 2 \delta}+g^{-1}(t)\right)\|f\|_{\mathbf{Y}} \\
& \leq C g^{-1}(t)\langle t\rangle^{-\rho / \delta-1 / \delta p}\|f\|_{\mathbf{Y}} .
\end{aligned}
$$

Similarly, by the third estimate of Lemma 2.1, we get for $s \in[0, \sigma], 1 \leq p \leq \infty$, and $t>0$,

$$
\begin{aligned}
\left\|\int_{0}^{t} g^{-1}(\tau) \mathscr{G}(t-\tau) f(\tau) d \tau\right\|_{\mathbf{B}^{s, p}} \\
\leq C \int_{0}^{t} e^{-(\mu / 2)(t-\tau)}\langle\tau\rangle^{-1-\gamma / \delta-1 / \delta p}\{t-\tau\}^{-s / v}\{\tau\}^{-\sigma / v} d \tau \\
\quad \times \sup _{\tau>0}\langle\tau\rangle^{1+\gamma / \delta+1 / \delta p}\{\tau\}^{\sigma / v}\|f(\tau)\|_{\mathbf{B}^{0, p}} \\
\quad \leq C\langle t\rangle^{-1-\gamma / \delta-1 / \delta p}\{t\}^{-s / v}\|f\|_{\mathbf{Y}} .
\end{aligned}
$$

Finally, by the fourth estimate of Lemma 2.1, we find for $\rho=0, \alpha, \beta, s \in[0, \sigma]$, and $t>0$,

$$
\begin{aligned}
&\left\|\int_{0}^{t} g^{-1}(\tau) \mathscr{G}(t-\tau) f(\tau) d \tau\right\|_{\mathbf{D}^{\rho, s}} \\
& \leq C \int_{0}^{t} d \tau\langle t-\tau\rangle^{-\rho / \delta}\{t-\tau\}^{-s / v} g^{-1}(\tau) \\
& \quad \times\left(\|f(\tau)\|_{\mathbf{D}^{0,0}}+\|f(\tau)\|_{\mathbf{A}^{0, \infty}}+\|f(\tau)\|_{\mathbf{B}^{0, \infty}}\right) \\
& \quad+C \int_{0}^{t} d \tau\langle t-\tau\rangle^{-\rho / \delta+\gamma / 2 \delta}\{t-\tau\}^{-s / v} g^{-1}(\tau) \\
& \quad \times\left(\|f(\tau)\|_{\mathbf{A}^{0, \infty}}+\|f(\tau)\|_{\mathbf{B}^{0, \infty}}\right)^{1 / 2}\|f(\tau)\|_{\mathbf{D}^{0,0}}^{1 / 2} .
\end{aligned}
$$

Using (2.39) and the norm Y, we have

$$
\begin{aligned}
& \left\|\int_{0}^{t} g^{-1}(\tau) \mathscr{G}(t-\tau) f(\tau) d \tau\right\|_{\mathbf{D}^{\rho, s}} \\
& \quad \leq C\|f\|_{\mathbf{Y}} \int_{0}^{\sqrt{t}}\langle t-\tau\rangle^{-\rho / \delta}\langle\tau\rangle^{\gamma / \delta-1}\{t-\tau\}^{-s / v}\{\tau\}^{-\sigma / v} d \tau \\
& \quad+C\|f\|_{\mathbf{Y}} \mathcal{g}^{-1}(t) \int_{\sqrt{t}}^{t}\langle t-\tau\rangle^{-\rho / \delta}\langle\tau\rangle^{\gamma / \delta-1}\{t-\tau\}^{-s / v}\{\tau\}^{-\sigma / v} d \tau
\end{aligned}
$$




$$
\begin{aligned}
& +C\|f\|_{\mathbf{Y}} \int_{0}^{\sqrt{t}}\langle t-\tau\rangle^{-\rho / \delta+\gamma / 2 \delta}\langle\tau\rangle^{\gamma / 2 \delta-1}\{t-\tau\}^{-s / v}\{\tau\}^{-\sigma / v} d \tau \\
& +C\|f\|_{\mathbf{Y}} g^{-1}(t) \int_{\sqrt{t}}^{t}\langle t-\tau\rangle^{-\rho / \delta+\gamma / 2 \delta}\langle\tau\rangle^{\gamma / 2 \delta-1}\{t-\tau\}^{-s / v}\{\tau\}^{-\sigma / v} d \tau \\
\leq & C\|f\|_{\mathbf{Y}}\{t\}^{-s / v}\langle t\rangle^{(\gamma-\rho) / \delta}\left(\langle t\rangle^{-\gamma / 4 \delta}+g^{-1}(t)\right) \\
\leq & C\|f\|_{\mathbf{Y}} g^{-1}(t)\{t\}^{-s / v}\langle t\rangle^{\gamma-\rho / \delta} .
\end{aligned}
$$

Whence the results of the lemma follow. Lemma 2.3 is proved.

Now we estimate the nonlinearity $\mathcal{N}(u)$ in the norms $\mathbf{A}^{0, p}, \mathbf{B}^{0, p}$, and $\mathbf{D}^{0,0}$.

LEMMA 2.4. Let the nonlinear operator $\mathcal{N}$ satisfy conditions (1.17) and (1.18). Then the inequalities

$$
\begin{aligned}
& \|\mathcal{N}(\varphi)\|_{\mathbf{A}^{0, p}} \leq C\left(\|\varphi(t)\|_{\mathbf{A}^{\alpha, 1}}+\|\varphi(t)\|_{\mathbf{B}^{\sigma, 1}}\right)\left(\|\varphi(t)\|_{\mathbf{A}^{0, p}}+\|\varphi(t)\|_{\mathbf{B}^{0, \infty}}\right) \\
& +C\left(\|\varphi(t)\|_{\mathbf{A}^{\beta, 1}}+\|\varphi(t)\|_{\mathbf{B}^{\sigma, 1}}\right)\left(\|\varphi(t)\|_{\mathbf{A}^{0,1}}+\|\varphi(t)\|_{\mathbf{B}^{0,1}}\right) \\
& \times\left(\|\varphi(t)\|_{\mathbf{A}^{0, p}}+\|\varphi(t)\|_{\mathbf{B}^{0, \infty}}\right), \\
& \|\mathcal{N}(\varphi)\|_{\mathbf{B}^{0, p}} \leq C\left(\|\varphi(t)\|_{\mathbf{A}^{\alpha+\gamma, 1}}+\|\varphi(t)\|_{\mathbf{B}^{\sigma, 1}}\right)\left(\|\varphi(t)\|_{\mathbf{A}^{0, p}}+\|\varphi(t)\|_{\mathbf{B}^{0, p}}\right) \\
& +C\left(\|\varphi(t)\|_{\mathbf{A}^{\alpha, 1}}+\|\varphi(t)\|_{\mathbf{B}^{\sigma, 1}}\right)\left(\|\varphi(t)\|_{\mathbf{A}^{\gamma, p}}+\|\varphi(t)\|_{\mathbf{B}^{0, p}}\right) \\
& +C\left(\|\varphi(t)\|_{\mathbf{A}^{\beta+\gamma}, 1}+\|\varphi(t)\|_{\mathbf{B}^{\sigma, 1}}\right)\left(\|\varphi(t)\|_{\mathbf{A}^{0, p}}+\|\varphi(t)\|_{\mathbf{B}^{0, p}}\right) \\
& \times\left(\|\varphi(t)\|_{\mathbf{A}^{0,1}}+\|\varphi(t)\|_{\mathbf{B}^{0,1}}\right) \\
& +C\left(\|\varphi(t)\|_{\mathbf{A}^{\beta, 1}}+\|\varphi(t)\|_{\mathbf{B}^{\sigma, 1}}\right)\left(\|\varphi(t)\|_{\mathbf{A}^{\gamma, p}}+\|\varphi(t)\|_{\mathbf{B}^{0, p}}\right) \\
& \times\left(\|\varphi(t)\|_{\mathbf{A}^{0,1}}+\|\varphi(t)\|_{\mathbf{B}^{0,1}}\right), \\
& \|\mathcal{N}(\varphi)\|_{\mathbf{D}^{0,0}} \leq C\|\varphi(t)\|_{\mathbf{D}^{\alpha, \sigma}}\left(\|\varphi(t)\|_{\mathbf{A}^{0,1}}+\|\varphi(t)\|_{\mathbf{B}^{0,1}}\right) \\
& +C\|\varphi(t)\|_{\mathbf{D}^{0,0}}\left(\|\varphi(t)\|_{\mathbf{A}^{\alpha, 1}}+\|\varphi(t)\|_{\mathbf{B}^{\sigma, 1}}\right) \\
& +C\left(\|\varphi(t)\|_{\mathbf{A}^{\alpha-\gamma, 1}}+\|\varphi(t)\|_{\mathbf{B}^{\sigma, 1}}\right)\left(\|\varphi(t)\|_{\mathbf{A}^{0, \infty}}+\|\varphi(t)\|_{\mathbf{B}^{0, \infty}}\right) \\
& +C\left(\|\varphi(t)\|_{\mathrm{A}^{-\gamma, 1}}+\|\varphi(t)\|_{\mathbf{B}^{0,1}}\right)\left(\|\varphi(t)\|_{\mathbf{A}^{\alpha, \infty}}+\|\varphi(t)\|_{\mathbf{B}^{\sigma, \infty}}\right) \\
& +C\|\varphi(t)\|_{\mathbf{D}^{\beta, \sigma}}\left(\|\varphi(t)\|_{\mathbf{A}^{0,1}}+\|\varphi(t)\|_{\mathbf{B}^{0,1}}\right)^{2} \\
& +C\|\varphi(t)\|_{\mathbf{D}^{0,0}}\left(\|\varphi(t)\|_{\mathbf{A}^{\beta, 1}}+\|\varphi(t)\|_{\mathbf{B}^{\sigma, 1}}\right) \\
& \times\left(\|\varphi(t)\|_{\mathbf{A}^{0,1}}+\|\varphi(t)\|_{\mathbf{B}^{0,1}}\right)
\end{aligned}
$$




$$
\begin{aligned}
& +C\left(\|\varphi(t)\|_{\mathrm{A}^{\beta-\gamma, 1}}+\|\varphi(t)\|_{\mathbf{B}^{\sigma, 1}}\right)\left(\|\varphi(t)\|_{\mathbf{A}^{0, \infty}}+\|\varphi(t)\|_{\mathbf{B}^{0, \infty}}\right) \\
& \times\left(\|\varphi(t)\|_{\mathbf{A}^{0,1}}+\|\varphi(t)\|_{\mathbf{B}^{0,1}}\right) \\
& +C\left(\|\varphi(t)\|_{\mathrm{A}^{-\gamma, 1}}+\|\varphi(t)\|_{\mathbf{B}^{0,1}}\right)\left(\|\varphi(t)\|_{\mathrm{A}^{\beta, \infty}}+\|\varphi(t)\|_{\mathbf{B}^{\sigma, \infty}}\right) \\
& \times\left(\|\varphi(t)\|_{\mathbf{A}^{0,1}}+\|\varphi(t)\|_{\mathbf{B}^{0,1}}\right)
\end{aligned}
$$

are valid for $1 \leq p \leq \infty$, provided that the right-hand sides are bounded.

Proof. By virtue of conditions (1.17) and (1.18), we obtain

$$
\begin{aligned}
\|\mathcal{N}(\varphi)\| \|_{\mathrm{A}^{0, p}} \leq & \left\|\int_{\mathbb{R}}|a(t, \xi, y)||\hat{\varphi}(t, \xi-y) \hat{\varphi}(t, y)| d y\right\|_{\mathbf{L}_{\xi}^{p}(|\xi| \leq 1)} \\
& +\left\|\int_{\mathbb{R}^{2}}|b(t, \xi, y, z)||\hat{\varphi}(t, \xi-y) \hat{\varphi}(t, y-z) \hat{\varphi}(t, z)| d y d z\right\|_{\mathbf{L}_{\xi}^{p}(|\xi| \leq 1)} \\
\leq & C\left\|\int_{\mathbb{R}}\left(\langle\xi-y\rangle^{\sigma}\{\xi-y\}^{\alpha}+\langle y\rangle^{\sigma}\{y\}^{\alpha}\right) \times|\hat{\varphi}(t, \xi-y) \hat{\varphi}(t, y)| d y\right\|_{\mathbf{L}_{\xi}^{p}(|\xi| \leq 1)} \\
& +C \| \int_{\mathbb{R}^{2}}\left(\langle\xi-y\rangle^{\sigma}\{\xi-y\}^{\beta}+\langle y-z\rangle^{\sigma}\{y-z\}^{\beta}+\langle z\rangle^{\sigma}\{z\}^{\beta}\right) \\
& \times|\hat{\varphi}(t, \xi-y) \hat{\varphi}(t, y-z) \hat{\varphi}(t, z)| d y d z \|_{L_{\xi}^{p}(|\xi| \leq 1)},
\end{aligned}
$$

and by the Young inequality, we have the estimate

$$
\begin{aligned}
& \left\|\int_{\mathbb{R}} \phi(\xi-y) \psi(y) d y\right\|_{\mathbf{L}_{\xi}^{p}(|\xi| \leq 1)} \\
& \quad \leq\left\|\int_{|y| \leq 1} \phi(\xi-y) \psi(y) d y\right\|_{\mathbf{L}_{\xi}^{p}(|\xi| \leq 1)}+\left\|\int_{|y|>1} \phi(\xi-y) \psi(y) d y\right\|_{\mathbf{L}_{\xi}^{\infty}(|\xi| \leq 1)} \\
& \quad \leq C\|\phi\|_{\mathbf{L}_{\xi}^{1}}\|\psi\|_{\mathbf{L}_{\xi}^{p}(|\xi| \leq 1)}+C\|\phi\|_{\mathbf{L}_{\xi}^{1}}\|\psi\|_{\mathbf{L}_{\xi}^{\infty}(|\xi|>1)},
\end{aligned}
$$

whence it follows that

$$
\begin{aligned}
\|\mathcal{N}(\varphi)\|_{\mathbf{A}^{0, p}} \leq & C\left\|\langle\xi\rangle^{\sigma}\{\xi\}^{\alpha} \hat{\varphi}(t, \xi)\right\|_{\mathbf{L}_{\xi}^{1}} \\
& \times\left(\|\hat{\varphi}(t, \xi)\|_{\mathbf{L}_{\xi}^{p}(|\xi| \leq 1)}+\|\hat{\varphi}(t, \xi)\|_{\mathbf{L}_{\xi}^{\infty}(|\xi|>1)}\right) \\
& +C\left\|\langle\xi\rangle^{\sigma}\{\xi\}^{\beta} \hat{\varphi}(t, \xi)\right\|_{\mathbf{L}_{\xi}^{1}} \\
& \times\left(\|\hat{\varphi}(t, \xi)\|_{\mathbf{L}_{\xi}^{p}(|\xi| \leq 1)}+\|\hat{\varphi}(t, \xi)\|_{\mathbf{L}_{\xi}^{\infty}(|\xi|>1)}\right)\|\hat{\varphi}(t, \xi)\|_{\mathbf{L}_{\xi}^{1}} \\
\leq & C\left(\|\varphi(t)\|_{\mathbf{A}^{\alpha, 1}}+\|\varphi(t)\|_{\mathbf{B}^{\sigma, 1}}\right)\left(\|\varphi(t)\|_{\mathbf{A}^{0, p}}+\|\varphi(t)\|_{\mathbf{B}^{0, \infty}}\right) \\
& +C\left(\|\varphi(t)\|_{\mathbf{A}^{\beta, 1}}+\|\varphi(t)\|_{\mathbf{B}^{\sigma, 1}}\right)\left(\|\varphi(t)\|_{\mathbf{A}^{0,1}}+\|\varphi(t)\|_{\mathbf{B}^{0,1}}\right) \\
& \times\left(\|\varphi(t)\|_{\mathbf{A}^{0, p}}+\|\varphi(t)\|_{\mathbf{B}^{0, \infty}}\right) .
\end{aligned}
$$


In the same manner,

$$
\begin{aligned}
\|\mathcal{N}(\varphi)\|_{\mathbf{B}^{0, p}} \leq & \left\|\int_{|y| \leq 1 / 2}|a(t, \xi, y)||\hat{\varphi}(t, \xi-y) \hat{\varphi}(t, y)| d y\right\|_{L_{\xi}^{p}(|\xi| \geq 1)} \\
& +\left\|\int_{|y| \geq 1 / 2}|a(t, \xi, y)||\hat{\varphi}(t, \xi-y) \hat{\varphi}(t, y)| d y\right\|_{L_{\xi}^{p}(|\xi| \geq 1)} \\
& +\| \int_{|y|+|z| \leq 1 / 2}|b(t, \xi, y, z)| \\
& \times|\hat{\varphi}(t, \xi-y) \hat{\varphi}(t, y-z) \hat{\varphi}(t, z)| d y d z \|_{L_{\xi}^{p}(|\xi| \geq 1)} \\
& +\| \int_{|y|+|z| \geq 1 / 2}|b(t, \xi, y, z)| \\
& \times|\hat{\varphi}(t, \xi-y) \hat{\varphi}(t, y-z) \hat{\varphi}(t, z)| d y d z \|_{L_{\xi}^{p}(|\xi| \geq 1)} .
\end{aligned}
$$

Whence

$$
\begin{aligned}
& \|\mathcal{N}(\varphi)\|_{\mathbf{B}^{0, p}=} \leq C \| \int_{|y| \leq 1 / 2}\{\xi-y\}^{\gamma}\left(\langle\xi-y\rangle^{\sigma}\{\xi-y\}^{\alpha}+\{y\}^{\alpha}\right) \\
& \times|\hat{\varphi}(t, \xi-y) \hat{\varphi}(t, y)| d y \|_{\mathbf{L}_{\xi}^{p}(|\xi| \geq 1)} \\
& +C \| \int_{|y| \geq 1 / 2}\{y\}^{\gamma}\left(\langle\xi-y\rangle^{\sigma}\{\xi-y\}^{\alpha}+\langle y\rangle^{\sigma}\{y\}^{\alpha}\right) \\
& \quad \times|\hat{\varphi}(t, \xi-y) \hat{\varphi}(t, y)| d y \|_{\mathbf{L}_{\xi}^{p}(|\xi| \geq 1)} \\
& +C \| \int_{|y|+|z| \leq 1 / 2}\{\xi-y\}^{\gamma}\left(\langle\xi-y\rangle^{\sigma}\{\xi-y\}^{\beta}+\{y-z\}^{\beta}+\{z\}^{\beta}\right) \\
& \quad \times|\hat{\varphi}(t, \xi-y) \hat{\varphi}(t, y-z) \hat{\varphi}(t, z)| d y d z \|_{\mathbf{L}_{\xi}^{p}(|\xi| \geq 1)} \\
& +C \| \int_{|y|+|z| \geq 1 / 2}\left(\{y-z\}^{\gamma}+\{z\}^{\gamma}\right) \\
& \quad \times\left(\langle\xi-y\rangle^{\sigma}\{\xi-y\}^{\beta}+\langle y-z\rangle^{\sigma}\{y-z\}^{\beta}+\langle z\rangle^{\sigma}\{z\}^{\beta}\right) \\
& \quad \times|\hat{\varphi}(t, \xi-y) \hat{\varphi}(t, y-z) \hat{\varphi}(t, z)| d y d z \|_{\mathbf{L}_{\xi}^{p}(|\xi| \geq 1)}
\end{aligned}
$$

Therefore,

$$
\begin{aligned}
\|\mathcal{N}(\varphi)\|_{\mathbf{B}^{0, p}} \leq & C\left\|\langle\xi\rangle^{\sigma}\{\xi\}^{\alpha+\gamma} \hat{\varphi}(t, \xi)\right\|_{\mathbf{L}_{\xi}^{1}}\|\hat{\varphi}(t, \xi)\|_{\mathbf{L}_{\xi}^{p}} \\
& +C\left\|\{\xi\}^{\gamma} \hat{\varphi}(t, \xi)\right\|_{\mathbf{L}_{\xi}^{p}}\left\|\langle\xi\rangle^{\sigma}\{\xi\}^{\alpha} \hat{\varphi}(t, \xi)\right\|_{\mathbf{L}_{\xi}^{1}} \\
\leq & C\left(\|\varphi(t)\|_{\mathbf{A}^{\alpha+\gamma, 1}}+\|\varphi(t)\|_{\mathbf{B}^{\sigma, 1}}\right)\left(\|\varphi(t)\|_{\mathbf{A}^{0, p}}+\|\varphi(t)\|_{\mathbf{B}^{0, p}}\right) \\
& +C\left(\|\varphi(t)\|_{\mathbf{A}^{\alpha, 1}}+\|\varphi(t)\|_{\mathbf{B}^{\sigma, 1}}\right)\left(\|\varphi(t)\|_{\mathbf{A}^{\gamma, p}}+\|\varphi(t)\|_{\mathbf{B}^{0, p}}\right)
\end{aligned}
$$




$$
\begin{aligned}
& +C\left(\|\varphi(t)\|_{\mathbf{A}^{\beta+\gamma, 1}}+\|\varphi(t)\|_{\mathbf{B}^{\sigma, 1}}\right)\left(\|\varphi(t)\|_{\mathbf{A}^{0, p}}+\|\varphi(t)\|_{\mathbf{B}^{0, p}}\right) \\
& \times\left(\|\varphi(t)\|_{\mathbf{A}^{0,1}}+\|\varphi(t)\|_{\mathbf{B}^{0,1}}\right) \\
& +C\left(\|\varphi(t)\|_{\mathbf{A}^{\beta, 1}}+\|\varphi(t)\|_{\mathbf{B}^{\sigma, 1}}\right)\left(\|\varphi(t)\|_{\mathbf{A}^{\gamma, p}}+\|\varphi(t)\|_{\mathbf{B}^{0, p}}\right) \\
& \times\left(\|\varphi(t)\|_{\mathbf{A}^{0,1}}+\|\varphi(t)\|_{\mathbf{B}^{0,1}}\right),
\end{aligned}
$$

whence the first two estimates of the lemma follow.

Denote

$$
\begin{aligned}
\tilde{a}(t, \xi, y)= & a(t, \xi, y)\left(\{\xi-y\}^{\alpha}\langle\xi-y\rangle^{\sigma}+\{y\}^{\alpha}\langle y\rangle^{\sigma}\right)^{-1}, \\
\Phi(t, \xi, y)= & \left(\{\xi-y\}^{\alpha}\langle\xi-y\rangle^{\sigma}+\{y\}^{\alpha}\langle y\rangle^{\sigma}\right) \hat{\varphi}(t, \xi-y) \hat{\varphi}(t, y), \\
\tilde{b}(t, \xi, y, z)= & b(t, \xi, y, z)\left(\{\xi-y\}^{\beta}\langle\xi-y\rangle^{\sigma}+\{y-z\}^{\beta}\langle y-z\rangle^{\sigma}+\{z\}^{\beta}\langle z\rangle^{\sigma}\right)^{-1}, \\
\Psi(t, \xi, y, z)= & \left(\{\xi-y\}^{\beta}\langle\xi-y\rangle^{\sigma}+\{y-z\}^{\beta}\langle y-z\rangle^{\sigma}+\{z\}^{\beta}\langle z\rangle^{\sigma}\right) \\
& \times \hat{\varphi}(t, \xi-y) \hat{\varphi}(t, y-z) \hat{\varphi}(t, z) .
\end{aligned}
$$

We have

$$
\begin{aligned}
\|\mathcal{N}(\varphi)\|_{D^{0,0}}= & \left\|\left|\partial_{\xi}\right|^{\gamma} \int_{\mathbb{R}} \tilde{a}(t, \xi, y) \Phi(t, \xi, y) d y\right\|_{L_{\xi}^{\infty}} \\
& +\left\|\left|\partial_{\xi}\right|^{\gamma} \int_{\mathbb{R}^{2}} \tilde{b}(t, \xi, y, z) \Psi(t, \xi, y, z) d y d z\right\|_{L_{\xi}^{\infty}} \\
\leq & C\left\|\int_{\mathbb{R}} \Phi(t, \xi, y)\left|\partial_{\xi}\right|^{\gamma} \tilde{a}(t, \xi, y) d y\right\|_{L_{\xi}^{\infty}} \\
& +C\left\|\int_{\mathbb{R}}\left[\left|\partial_{\xi}\right|^{\gamma}, \Phi(t, \xi, y)\right] \tilde{a}(t, \xi, y) d y\right\|_{L_{\xi}^{\infty}} \\
& +C\left\|\int_{\mathbb{R}^{2}} \Psi(t, \xi, y, z)\left|\partial_{\xi}\right|^{\gamma} \tilde{b}(t, \xi, y, z) d y d z\right\|_{L_{\xi}^{\infty}} \\
& +C\left\|\int_{\mathbb{R}^{2}}\left[|\partial \xi|^{\gamma}, \Psi(t, \xi, y, z)\right] \tilde{b}(t, \xi, y, z) d y d z\right\|_{L_{\xi}^{\infty}},
\end{aligned}
$$

where the commutators

$$
\begin{aligned}
& {\left[\left|\partial_{\xi}\right|^{\gamma}, \Phi(t, \xi, y)\right] \tilde{a}(t, \xi, y)} \\
& \quad \equiv \int_{\mathbb{R}}|\Phi(t, \xi-\eta, y)-\Phi(t, \xi, y)| \tilde{a}(t, \xi-\eta, y)|\eta|^{-1-\gamma} d \eta,
\end{aligned}
$$

and similarly,

$$
\begin{aligned}
& {\left[\left|\partial_{\xi}\right|^{\gamma}, \Psi(t, \xi, y, z)\right] \tilde{b}(t, \xi, y, z)} \\
& \quad \equiv \int_{\mathbb{R}}|\Psi(t, \xi-\eta, y, z)-\Psi(t, \xi, y, z)| \tilde{b}(t, \xi-\eta, y, z)|\eta|^{-1-\gamma} d \eta .
\end{aligned}
$$


By virtue of condition (1.17), we estimate the commutator

$$
\begin{aligned}
\left\|\int_{\mathbb{R}}\left[\left|\partial_{\xi}\right|^{\gamma}, \Phi(t, \xi, y)\right] \tilde{a}(t, \xi, y) d y\right\|_{\mathbf{L}_{\xi}^{\infty}} \\
\leq C\left\|\int_{\mathbb{R}} \int_{\mathbb{R}}|\Phi(t, \xi-\eta, y)-\Phi(t, \xi, y)||\eta|^{-1-\gamma} d \eta d y\right\|_{\mathbf{L}_{\xi}^{\infty}} \\
\leq C\left\|\int_{\mathbb{R}}\left(\left|\partial_{\xi}\right|^{\gamma}\{\xi-y\}^{\alpha}\langle\xi-y\rangle^{\sigma} \hat{\varphi}(t, \xi-y)\right) \hat{\varphi}(t, y) d y\right\|_{\mathbf{L}_{\xi}^{\infty}} \\
\quad+C\left\|\int_{\mathbb{R}}\left(\left|\partial_{\xi}\right|^{\gamma} \hat{\varphi}(t, \xi-y)\right)\{y\}^{\alpha}\langle y\rangle^{\sigma} \hat{\varphi}(t, y) d y\right\|_{\mathbf{L}_{\xi}^{\infty}} \\
\leq C\left\|\left|\partial_{\xi}\right|^{\gamma}\{\xi\}^{\alpha}\langle\xi\rangle^{\sigma} \hat{\varphi}(t, \xi)\right\|_{\mathbf{L}_{\xi}^{\infty}}\|\hat{\varphi}(t, \xi)\|_{\mathbf{L}_{\xi}^{1}} \\
\quad+C\left\|\left|\partial_{\xi}\right|^{\gamma} \hat{\varphi}(t, \xi)\right\|_{\mathbf{L}_{\xi}^{\infty}}\left\|\{\xi\}^{\alpha}\langle\xi\rangle^{\sigma} \hat{\varphi}(t, \xi)\right\|_{\mathbf{L}_{\xi}^{1}} \\
\leq C\|\varphi(t)\|_{\mathbf{D}^{\alpha, \sigma}}\left(\|\varphi(t)\|_{\mathbf{A}^{0,1}}+\|\varphi(t)\|_{\mathbf{B}^{0,1}}\right) \\
\quad+C\|\varphi(t)\|_{\mathbf{D}^{0,0}}\left(\|\varphi(t)\|\left\|_{\mathbf{A}^{\alpha, 1}}+\right\| \varphi(t) \|_{\mathbf{B}^{\sigma, 1}}\right) .
\end{aligned}
$$

In the same manner, by (1.18), we get

$$
\begin{aligned}
\| \int_{\mathbb{R}^{2}}[ & \left.|\partial \xi|^{\gamma}, \Psi(t, \xi, y, z)\right] \tilde{b}(t, \xi, y, z) d y d z \|_{\mathbf{L}_{\xi}^{\infty}} \\
\leq & C\|\varphi(t)\|_{\mathbf{D}^{\beta, \sigma}}\left(\|\varphi(t)\|_{\mathbf{A}^{0,1}}+\|\varphi(t)\|_{\mathbf{B}^{0,1}}\right)^{2} \\
& +C\|\varphi(t)\|_{\mathbf{D}^{0,0}}\left(\|\varphi(t)\|_{\mathbf{A}^{\beta, 1}}+\|\varphi(t)\|_{\mathbf{B}^{\sigma, 1}}\right) \\
& \times\left(\|\varphi(t)\|_{\mathbf{A}^{0,1}}+\|\varphi(t)\|_{\mathbf{B}^{0,1}}\right) .
\end{aligned}
$$

Via (1.17), we have

$$
\begin{aligned}
& \int_{|\eta| \geq(1 / 2)\{\xi-y\}}|\tilde{a}(t, \xi-\eta, y)-\tilde{a}(t, \xi, y)| \frac{d \eta}{|\eta|^{1+\gamma}} \leq C \int_{|\eta| \geq(1 / 2)\{\xi-y\}} \frac{d \eta}{|\eta|^{1+\gamma}} \leq C\{\xi-y\}^{-\gamma} \\
& \int_{|\eta| \leq(1 / 2)\{\xi-y\}}|\tilde{a}(t, \xi-\eta, y)-\tilde{a}(t, \xi, y)| \frac{d \eta}{|\eta|^{1+\gamma}} \\
& \leq C \int_{|\eta| \leq(1 / 2)\{\xi-y\}}\left|\int_{\xi-y}^{\xi-y-\eta}\{\zeta\}^{-1} d \zeta\right| \frac{d \eta}{|\eta|^{1+\gamma}} \\
& \leq C\{\xi-y\}^{-1} \int_{|\eta| \leq(1 / 2)\{\xi-y\}} \frac{d \eta}{|\eta|^{\gamma}} \leq C\{\xi-y\}^{-\gamma}
\end{aligned}
$$

Thus, we have the estimate

$$
\left|\partial_{\xi}\right|^{\gamma} \tilde{a}(t, \xi, y)=\int_{\mathbb{R}}|\tilde{a}(t, \xi-\eta, y)-\tilde{a}(t, \xi, y)||\eta|^{-1-\gamma} d \eta \leq C\{\xi-y\}^{-\gamma}
$$


for all $\xi, y \in \mathbb{R}$. Therefore,

$$
\begin{aligned}
\| \int_{\mathbb{R}} \Phi & (t, \xi, y)\left|\partial_{\xi}\right|^{\gamma} \tilde{a}(t, \xi, y) d y \|_{\mathbf{L}_{\xi}^{\infty}} \\
\leq & C\left\|\int_{\mathbb{R}}\{\xi-y\}^{\alpha-\gamma}\langle\xi-y\rangle^{\sigma} \hat{\varphi}(t, \xi-y) \hat{\varphi}(t, y) d y\right\|_{\mathbf{L}_{\xi}^{\infty}} \\
& +C\left\|\int_{\mathbb{R}}\{\xi-y\}^{-\gamma} \hat{\varphi}(t, \xi-y)\{y\}^{\alpha}\langle y\rangle^{\sigma} \hat{\varphi}(t, y) d y\right\|_{\mathbf{L}_{\xi}^{\infty}} \\
\leq & C\left\|\{\xi\}^{\alpha-\gamma}\langle\xi\rangle^{\sigma} \hat{\varphi}(t, \xi)\right\|_{\mathbf{L}_{\xi}^{1}}\|\hat{\varphi}(t, \xi)\|_{\mathbf{L}_{\xi}^{\infty}} \\
& +C\left\|\{\xi\}^{-\gamma} \hat{\varphi}(t, \xi)\right\|_{\mathbf{L}_{\xi}^{1}}\left\|\{\xi\}^{\alpha}\langle\xi\rangle^{\sigma} \hat{\varphi}(t, \xi)\right\|_{\mathbf{L}_{\xi}^{\infty}} \\
\leq & C\left(\left.\|\varphi(t)\|\right|_{\mathbf{A}^{\alpha-\gamma, 1}}+\|\varphi(t)\|_{\mathbf{B}^{\sigma, 1}}\right)\left(\|\varphi(t)\|_{\mathbf{A}^{0, \infty}}+\|\varphi(t)\|_{\mathbf{B}^{0, \infty}}\right) \\
& +C\left(\|\varphi(t)\|_{\mathbf{A}^{-\gamma, 1}}+\|\varphi(t)\|_{\mathbf{B}^{0,1}}\right)\left(\|\varphi(t)\|_{\mathbf{A}^{\alpha, \infty}}+\|\varphi(t)\|_{\mathbf{B}^{\sigma, \infty}}\right) .
\end{aligned}
$$

Similarly, we obtain

$$
\begin{aligned}
\| \int_{\mathbb{R}^{2}} \Psi & (t, \xi, y, z)\left|\partial_{\xi}\right|^{\gamma} \tilde{b}(t, \xi, y, z) d y d z \|_{\mathbf{L}_{\xi}^{\infty}} \\
\leq & C\left(\|\varphi(t)\|_{\mathbf{A}^{\beta-\gamma, 1}}+\|\varphi(t)\|_{\mathbf{B}^{\sigma, 1}}\right)\left(\|\varphi(t)\|_{\mathbf{A}^{0, \infty}}+\|\varphi(t)\|_{\mathbf{B}^{0, \infty}}\right) \\
& \times\left(\|\varphi(t)\|_{\mathbf{A}^{0,1}}+\|\varphi(t)\|_{\mathbf{B}^{0,1}}\right) \\
& +C\left(\|\varphi(t)\|_{\mathrm{A}^{-\gamma, 1}}+\|\varphi(t)\|_{\mathbf{B}^{0,1}}\right)\left(\|\varphi(t)\|_{\mathrm{A}^{\beta, \infty}}+\|\varphi(t)\|_{\mathbf{B}^{\sigma, \infty}}\right) \\
& \times\left(\|\varphi(t)\|_{\mathbf{A}^{0,1}}+\|\varphi(t)\|_{\mathbf{B}^{0,1}}\right) .
\end{aligned}
$$

In view of (2.52), (2.55), (2.56), (2.59), and (2.60), we get

$$
\begin{aligned}
\|\mathcal{N}(\varphi)\|_{\mathbf{D}^{0,0}} \leq & C\|\varphi(t)\|_{\mathbf{D}^{\alpha, \sigma}}\left(\|\varphi(t)\|_{\mathbf{A}^{0,1}}+\|\varphi(t)\|_{\mathbf{B}^{0,1}}\right) \\
& +C\|\varphi(t)\|_{\mathbf{D}^{0,0}}\left(\|\varphi(t)\|_{\mathbf{A}^{\alpha, 1}}+\|\varphi(t)\|_{\mathbf{B}^{\sigma, 1}}\right) \\
& +C\left(\|\varphi(t)\|_{\mathbf{A}^{\alpha-\gamma, 1}}+\|\varphi(t)\|_{\mathbf{B}^{\sigma, 1}}\right)\left(\|\varphi(t)\|_{\mathbf{A}^{0, \infty}}+\|\varphi(t)\|_{\mathbf{B}^{0, \infty}}\right) \\
& +C\left(\|\varphi(t)\|_{\mathbf{A}^{-\gamma, 1}}+\|\varphi(t)\|_{\mathbf{B}^{0,1}}\right)\left(\|\varphi(t)\|_{\mathbf{A}^{\alpha, \infty}}+\|\varphi(t)\|_{\mathbf{B}^{\sigma, \infty}}\right) \\
& +C\|\varphi(t)\|_{\mathbf{D}^{\beta, \sigma}}\left(\|\varphi(t)\|_{\mathbf{A}^{0,1}}+\|\varphi(t)\|_{\mathbf{B}^{0,1}}\right)^{2} \\
& +C\|\varphi(t)\|_{\mathbf{D}^{0,0}}\left(\|\varphi(t)\|_{\mathbf{A}^{\beta, 1}}+\|\varphi(t)\|_{\mathbf{B}^{\sigma, 1}}\right) \\
& \times\left(\|\varphi(t)\|_{\mathbf{A}^{0,1}}+\|\varphi(t)\|_{\mathbf{B}^{0,1}}\right) \\
& +C\left(\|\varphi(t)\|_{\mathbf{A}^{\beta-\gamma, 1}}+\|\varphi(t)\|_{\mathbf{B}^{\sigma, 1}}\right)\left(\|\varphi(t)\|_{\mathbf{A}^{0, \infty}}+\|\varphi(t)\|_{\mathbf{B}^{0, \infty}}\right) \\
& \times\left(\|\varphi(t)\|_{\mathbf{A}^{0,1}}+\|\varphi(t)\|_{\mathbf{B}^{0,1}}\right) \\
& +C\left(\|\varphi(t)\|_{\mathbf{A}^{-\gamma, 1}}+\|\varphi(t)\|_{\mathbf{B}^{0,1}}\right)\left(\|\varphi(t)\|_{\mathbf{A}^{\beta, \infty}}+\|\varphi(t)\|_{\mathbf{B}^{\sigma, \infty}}\right) \\
& \times\left(\|\varphi(t)\|_{\mathbf{A}^{0,1}}+\|\varphi(t)\|_{\mathbf{B}^{0,1}}\right) .
\end{aligned}
$$

Thus, the third estimate of the lemma is true. Lemma 2.4 is proved. 
The next lemma will be used in the proof of Theorem 1.1 to evaluate large-time behavior of the mean value of the nonlinearity in (1.1) in the norms $\mathbf{A}^{0, p}, \mathbf{B}^{0, p}$, and $\mathbf{D}^{0,0}$. We use the notations

$$
\begin{aligned}
\mathcal{N}_{0}(\varphi)= & \overline{\mathscr{F}}_{\xi \rightarrow x} \int_{\mathbb{R}} a_{0}(y) \hat{\varphi}(t, \xi-y) \hat{\varphi}(t, y) d y \\
& +\omega \overline{\mathscr{F}} \xi \rightarrow x \int_{\mathbb{R}^{2}} b_{0}(y, z) \hat{\varphi}(t, \xi-y) \hat{\varphi}(t, y-z) \hat{\varphi}(t, z) d y d z,
\end{aligned}
$$

where $\omega=0$ if $a_{0} \neq 0$ and $\omega=1$ if $a_{0} \equiv 0$. We also define as above

$$
\begin{aligned}
\varkappa \equiv & \theta^{2} \int_{\mathbb{R}} a_{0}(y) e^{-L_{0}(-y)-L_{0}(y)} d y \\
& +\omega \theta^{3} \int_{\mathbb{R}^{2}} b_{0}(y, z) e^{-L_{0}(-y)-L_{0}(y-z)-L_{0}(z)} d y d z>0,
\end{aligned}
$$

where $\theta=\hat{u}_{0}(0)$ and $g(t)=1+\varkappa \log \langle t\rangle$.

LEMMA 2.5. Let the linear operator $\mathscr{L}$ satisfy conditions (1.14) and (1.16) and the nonlinear operator $\mathcal{N}$ satisfy conditions (1.17), (1.18), (1.21), and (1.22). Assume that $u_{0}$ is such that the norm $\left\|u_{0}\right\|_{\mathbf{A}^{0, \infty}}+\left\|u_{0}\right\|_{\mathbf{D}^{0,0}}=\varepsilon$. Let the function $v(t, x)$ satisfy the estimates

$$
\begin{aligned}
\|v\|_{\mathrm{X}} & \leq C \varepsilon \\
\left\|v(t)-G(t) u_{0}\right\|_{\mathrm{A}^{\rho, p}} & \leq C \varepsilon^{2} g^{-1}(t)\langle t\rangle^{-\rho / \delta-1 / \delta p},
\end{aligned}
$$

where $\rho \in[0, \alpha]$ and $1 \leq p \leq \infty$.

Then the inequalities

$$
\left|1+\int_{0}^{t} \widehat{\mathcal{N}_{1}(v)}(\tau, 0) d \tau-\varkappa \log t\right| \leq \frac{C \varepsilon^{3}}{\varkappa} \log (g(t))+C \varepsilon^{2}
$$

if $a_{0} \neq 0$ and

$$
\left|1+\int_{0}^{t} \widehat{\mathcal{N}_{2}(v)}(\tau, 0) d \tau-\varkappa \log t\right| \leq \frac{C \varepsilon^{4}}{\varkappa} \log (g(t))+C \varepsilon^{3}
$$

if $a_{0} \equiv 0$ are valid for all $t>0$.

Proof. By Lemma 2.4 and in view of the condition (2.64), we get

$$
\begin{aligned}
\left|\int_{0}^{t} \widehat{\mathcal{N}(v)}(\tau, 0) d \tau\right| & \leq C \int_{0}^{t}\|\mathcal{N}(v(\tau))\|_{\mathrm{A}^{0, \infty}} d \tau \\
& \leq C\|v\|_{\mathrm{X}}^{2} \int_{0}^{t}\{\tau\}^{-\sigma / v} d \tau \\
& \leq C \varepsilon^{2} t^{1-\sigma / v},
\end{aligned}
$$


whence estimates (2.66) and (2.67) follow for all $0<t<1$. We now consider $t \geq 1$. By Lemma 2.2 and via the condition of the lemma, we get

$$
\begin{aligned}
\| v(\tau) & -\theta \tau^{-1 / \delta} G_{0}\left(\tau^{-1 / \delta}(\cdot)\right) \|_{\mathrm{A}^{\rho, p}} \\
\leq & C\left\|v(\tau)-\mathscr{G}(\tau) u_{0}\right\|_{\mathrm{A}^{\rho, p}} \\
& +\left\|\mathscr{G}(\tau) u_{0}-\theta \tau^{-1 / \delta} G_{0}\left(\tau^{-1 / \delta}(\cdot)\right)\right\|_{\mathrm{A}^{\rho, p}} \\
\leq & C \varepsilon^{2} g^{-1}(\tau)\langle\tau\rangle^{-\rho / \delta-1 / \delta p}+C\langle\tau\rangle^{-(\rho+\gamma) / \delta-1 / \delta p}\left\|u_{0}\right\|_{\mathrm{A}^{0, \infty}} \\
& +C\langle\tau\rangle^{-(\rho+\gamma) / \delta-1 / \delta p}\left\|u_{0}\right\|_{\mathbf{D}^{0,0}} \\
\leq & C \varepsilon\langle\tau\rangle^{-\rho / \delta-1 / \delta p}\left(\varepsilon g^{-1}(\tau)+\langle\tau\rangle^{-\gamma / \delta}+\langle\tau\rangle^{-\gamma / \delta}\right)
\end{aligned}
$$

for all $\tau>0$, where $1 \leq p \leq \infty$ and $\rho \geq 0$. By condition (1.17), we get

$$
\begin{aligned}
& \left|\int_{|y| \leq 1}\left(a(\tau, 0, y)-a_{0}(y)\right) \hat{v}(\tau,-y) \hat{v}(\tau, y) d y\right| \\
& \quad \leq C \int_{|y| \leq 1}|y|^{\alpha+\gamma}|\hat{v}(\tau,-y)||\hat{v}(\tau, y)| d y \\
& \quad \leq C\|v(\tau)\|_{\mathrm{A}^{\alpha, 1}}\|v(\tau)\|_{\mathrm{A}^{\gamma, \infty}} \\
& \quad \leq C \varepsilon^{2}\{\tau\}^{-\sigma / v}\langle\tau\rangle^{-1-\gamma / \delta}
\end{aligned}
$$

for all $\tau>0$. In the same manner, via (1.18), we obtain

$$
\begin{aligned}
& \left|\int_{|y|+|z| \leq 1}\left(b(\tau, 0, y, z)-b_{0}(y, z)\right) \hat{v}(\tau,-y) \hat{v}(\tau, y-z) \hat{v}(\tau, z) d y d z\right| \\
& \quad \leq C \varepsilon^{3}\{\tau\}^{-\sigma / v}\langle\tau\rangle^{-1-\gamma / \delta} .
\end{aligned}
$$

Further we find

$$
\begin{aligned}
& \left|\widehat{\mathcal{N}_{1}(v)}(\tau, 0)-\mathscr{F}_{x \rightarrow \xi}\left(\mathcal{N}_{0}\left(\theta \tau^{-1 / \delta} G_{0}\left(x \tau^{-1 / \delta}\right)\right)\right)(\tau, 0)\right| \\
& \leq\left|\int_{|y| \leq 1}\left(a(\tau, 0, y)-a_{0}(y)\right) \hat{v}(\tau,-y) \hat{v}(\tau, y) d y\right| \\
& \quad+\left|\int_{|y| \leq 1} a_{0}(y)\left(\hat{v}(\tau,-y) \hat{v}(\tau, y)-\theta^{2} e^{-\tau L_{0}(-y)-\tau L_{0}(y)}\right) d y\right| \\
& \quad+\left|\int_{|y| \geq 1} a(\tau, 0, y) \hat{v}(\tau,-y) \hat{v}(\tau, y) d y\right| \\
& \quad+\theta^{2}\left|\int_{|y| \geq 1} a_{0}(y) e^{-\tau L_{0}(-y)-\tau L_{0}(y)} d y\right|
\end{aligned}
$$


when $a_{0}(y) \neq 0$, and if $a_{0}(y) \equiv 0$, we have

$$
\begin{aligned}
& \left|\widehat{\mathcal{N}_{2}(v)}(\tau, 0)-\mathscr{F}_{x \rightarrow \xi}\left(\mathcal{N}_{0}\left(\theta \tau^{-1 / \delta} G_{0}\left(x \tau^{-1 / \delta}\right)\right)\right)(\tau, 0)\right| \\
& \leq\left|\int_{|y|+|z| \leq 1}\left(b(\tau, 0, y, z)-b_{0}(y, z)\right) \hat{v}(\tau,-y) \hat{v}(\tau, y-z) \hat{v}(\tau, z) d y\right| \\
& \quad+\mid \int_{|y|+|z| \leq 1} b_{0}(y, z)(\hat{v}(\tau,-y) \hat{v}(\tau, y-z) \hat{v}(\tau, z) \\
& \left.\quad-\theta^{3} e^{-\tau L_{0}(-y)-\tau L_{0}(y-z)-\tau L_{0}(z)}\right) d y d z \mid \\
& \quad+\left|\int_{|y|+|z| \geq 1} b(\tau, 0, y, z) \hat{v}(\tau,-y) \hat{v}(\tau, y-z) \hat{v}(\tau, z) d y d z\right| \\
& \quad+\theta^{3}\left|\int_{|y|+|z| \geq 1} b_{0}(y, z) e^{-\tau L_{0}(-y)-\tau L_{0}(y-z)-\tau L_{0}(z)} d y d z\right|
\end{aligned}
$$

whence, applying (2.69), (2.70), and (2.71), we obtain

$$
\begin{aligned}
\left|\widehat{\mathcal{N}_{1}(v)}(\tau, 0)-\mathscr{F}_{x \rightarrow \xi}\left(\mathcal{N}_{0}\left(\theta \tau^{-1 / \delta} G_{0}\left(x \boldsymbol{T}^{-1 / \delta}\right)\right)\right)(\tau, 0)\right| \\
\leq C \varepsilon^{2}\{\boldsymbol{\tau}\}^{-\sigma / v}\langle\tau\rangle^{-1-\gamma / \delta}+C\left\|v(\boldsymbol{\tau})-\theta \tau^{-1 / \delta} G_{0}\left(\tau^{-1 / \delta}(\cdot)\right)\right\|_{\mathbf{A}^{0, \infty}} \\
\quad \times\left(\|v(\boldsymbol{\tau})\|_{\mathbf{A}^{\alpha, 1}}+\left\|\theta \boldsymbol{\tau}^{-1 / \delta} G_{0}\left(\tau^{-1 / \delta}(\cdot)\right)\right\|_{\mathbf{A}^{\alpha, 1}}\right) \\
\quad+C\|v(\boldsymbol{\tau})\|_{\mathbf{B}^{\sigma, 1}}\|v(\boldsymbol{\tau})\|_{\mathbf{B}^{0, \infty}} \\
\quad+C \theta^{2}\left\|\boldsymbol{\tau}^{-1 / \delta} G_{0}\left(\tau^{-1 / \delta}(\cdot)\right)\right\|_{\mathbf{B}^{\alpha, 1}}\left\|\boldsymbol{\tau}^{-1 / \delta} G_{0}\left(\tau^{-1 / \delta}(\cdot)\right)\right\|_{\mathbf{B}^{0, \infty}} .
\end{aligned}
$$

Thus,

$$
\begin{aligned}
\left|\widehat{\mathcal{N}_{1}(v)}(\tau, 0)-\mathscr{F}_{x \rightarrow \xi}\left(\mathcal{N}_{0}\left(\theta \tau^{-1 / \delta} G_{0}\left(x \tau^{-1 / \delta}\right)\right)\right)(\tau, 0)\right| \\
\leq C \varepsilon^{2}\{\tau\}^{-\sigma / v}\langle\tau\rangle^{-1-\gamma / \delta} \\
\quad+C \varepsilon^{2}\langle\tau\rangle^{-1}\left(\varepsilon g^{-1}(\tau)+\langle\tau\rangle^{-\gamma / \delta}+\langle\tau\rangle^{-\gamma / \delta}\right) \\
\quad+C \varepsilon^{2}\{t\}^{-\sigma / v}\langle t\rangle^{-1-\gamma / \delta}+C \varepsilon^{2}\{t\}^{-\alpha / \delta}\langle t\rangle^{-1-\gamma / \delta} .
\end{aligned}
$$

In the same manner, we get

$$
\begin{aligned}
\left|\widehat{\mathcal{N}_{2}(v)}(\tau, 0)-\mathscr{F}_{x \rightarrow \xi}\left(\mathcal{N}_{0}\left(\theta \tau^{-1 / \delta} G_{0}\left(x \tau^{-1 / \delta}\right)\right)\right)(\tau, 0)\right| \\
\leq C \varepsilon^{3}\{\tau\}^{-\sigma / v}\langle\tau\rangle^{-1-\gamma / \delta} \\
\quad+C \varepsilon^{3}\langle\tau\rangle^{-1}\left(\varepsilon g^{-1}(\tau)+\langle\tau\rangle^{-\gamma / \delta}+\langle\tau\rangle^{-\gamma / \delta}\right) \\
\quad+C \varepsilon^{3}\{t\}^{-\sigma / v}\langle t\rangle^{-1-\gamma / \delta}+C \varepsilon^{3}\{t\}^{-\beta / \delta}\langle t\rangle^{-1-\gamma / \delta}
\end{aligned}
$$


for all $\tau>0$. By an explicit computation, we have

$$
\begin{aligned}
\mathscr{F}_{x \rightarrow \xi} & \left(\mathcal{N}_{0}\left(\theta \tau^{-1 / \delta} G_{0}\left(x \tau^{-1 / \delta}\right)\right)\right)(\tau, 0) \\
= & \theta^{2} \int_{\mathbb{R}} a_{0}(y) e^{-\tau L_{0}(-y)-\tau L_{0}(y)} d y \\
& +\omega \theta^{3} \int_{\mathbb{R}^{2}} b_{0}(y, z) e^{-\tau L_{0}(-y)-\tau L_{0}(y-z)-\tau L_{0}(z)} d y d z \\
= & \theta^{2} \tau^{-1} \int_{\mathbb{R}} a_{0}(y) e^{-L_{0}(-y)-L_{0}(y)} d y \\
& +\omega \theta^{3} \tau^{-1} \int_{\mathbb{R}^{2}} b_{0}(y, z) e^{-L_{0}(-y)-L_{0}(y-z)-L_{0}(z)} d y d z \\
= & \varkappa \tau^{-1},
\end{aligned}
$$

where $\omega=0$ if $a_{0} \neq 0$ and $\omega=1$ if $a_{0} \equiv 0$. Therefore, we obtain

$$
\begin{aligned}
\mid \int_{1}^{t} & \widehat{\mathcal{N}_{1}(v)}(\tau, 0) d \tau-\varkappa \log t \mid \\
\leq & C \varepsilon^{3} \int_{1}^{t} \frac{d \tau}{\tau(1+\varkappa \log \langle t\rangle)} \\
& +C \varepsilon^{2} \int_{1}^{t} \tau^{-1-\gamma / \delta} d \tau+C \varepsilon^{2} \int_{1}^{t} \tau^{-1-\gamma / \delta} d \tau \\
\leq & \frac{C \varepsilon^{3}}{\varkappa} \log (1+\varkappa \log \langle t\rangle)+C \varepsilon^{2}
\end{aligned}
$$

for all $t \geq 1$ if $a_{0} \neq 0$ and

$$
\begin{aligned}
\left|\int_{1}^{t} \widehat{\mathcal{N}(v)}(\tau, 0) d \tau-\varkappa \log t\right| \leq & C \varepsilon^{4} \int_{1}^{t} \frac{d \tau}{\tau(1+\varkappa \log \langle t\rangle)} \\
& +C \varepsilon^{3} \int_{1}^{t} \tau^{-1-\gamma / \delta} d \tau+C \varepsilon^{3} \int_{1}^{t} \tau^{-1-\gamma / \delta} d \tau \\
\leq & \frac{C \varepsilon^{4}}{\varkappa} \log (1+\varkappa \log \langle t\rangle)+C \varepsilon^{3}
\end{aligned}
$$

for all $t \geq 1$ if $a_{0} \equiv 0$. Whence in view of (2.68), the result of the lemma follows. Lemma 2.5 is proved.

3. Proof of Theorem 1.1. For the local existence of classical solutions for the Cauchy problem (1.1), we refer to [2]. We make a change of dependent variable $u(t, x)=$ $e^{-\varphi(t)} v(t, x)$, then we get from (1.1)

$$
v_{t}+\mathscr{L} v+e^{-\varphi(t)} \mathcal{N}_{1}(v)+e^{-2 \varphi(t)} \mathcal{N}_{2}(v)-\varphi^{\prime} v=0,
$$

where

$$
\begin{gathered}
\mathcal{N}_{1}(u)=\overline{\mathscr{F}_{\xi} \rightarrow x} \int_{\mathbb{R}} a(t, \xi, y) \hat{u}(t, \xi-y) \hat{u}(t, y) d y, \\
\mathcal{N}_{2}(u)=\overline{\mathscr{F}} \xi \rightarrow x \int_{\mathbb{R}^{2}} b(t, \xi, y, z) \hat{u}(t, \xi-y) \hat{u}(t, y-z) \hat{u}(t, z) d y d z .
\end{gathered}
$$


Now, we require that the real-valued function $\varphi(t)$ satisfies the following condition expressed in terms of the Fourier transform:

$$
e^{-\varphi(t)} \widehat{\mathcal{N}_{1}}(v)(t, 0)+e^{-2 \varphi(t)} \widehat{\mathcal{N}_{2}}(v)(t, 0)-\varphi^{\prime} \widehat{v}(t, 0)=0,
$$

whence, via (3.1), we get

$$
\frac{d}{d t} \hat{v}(t, 0)=0
$$

for all $t>0$. Therefore,

$$
e^{-\varphi(t)} \widehat{\mathcal{N}_{1}}(v)(t, 0)+e^{-2 \varphi(t)} \widehat{\mathcal{N}_{2}}(v)(t, 0)=\varphi^{\prime} \hat{v}(0,0) .
$$

If we choose the initial condition $\varphi(0)=0$, we have

$$
\hat{v}(t, 0)=\hat{v}(0,0)=e^{\varphi(0)} \hat{u}_{0}(0)=\theta
$$

and we obtain the following system:

$$
\begin{gathered}
v_{t}+\mathscr{L} v=-e^{-\varphi(t)}\left(\mathcal{N}_{1}(v)-\frac{v}{\theta} \widehat{\mathcal{N}_{1}}(v)(t, 0)\right) \\
-e^{-2 \varphi(t)}\left(\mathcal{N}_{2}(v)-\frac{v}{\theta} \widehat{\mathcal{N}_{2}}(v)(t, 0)\right), \\
\varphi^{\prime}(t)=\frac{1}{\theta} e^{-\varphi(t)} \widehat{\mathcal{N}_{1}}(v)(t, 0)+\frac{1}{\theta} e^{-2 \varphi(t)} \widehat{\mathcal{N}_{2}}(v)(t, 0), \\
v(0, x)=u_{0}(x), \quad \varphi(0)=0 .
\end{gathered}
$$

Multiplying the second equation of system (3.7) by the factor $e^{\varphi(t)}$ then integrating with respect to time $t>0$ and making a change of the dependent variables $v=\mathscr{G}(t) u_{0}+r$ and $e^{\varphi(t)}=h_{1}(t)$, we get the system of integral equations

$$
\begin{gathered}
r=-\int_{0}^{t} h_{1}^{-1}(\tau) \mathscr{G}(t-\tau) f_{1}(\tau) d \tau, \\
h_{1}=1+\frac{1}{\theta} \int_{0}^{t}\left(\widehat{\mathcal{N}_{1}}(v)+\frac{1}{h_{1}(\tau)} \widehat{\mathcal{N}_{2}}(v)\right)(\tau, 0) d \tau,
\end{gathered}
$$

where

$$
\begin{aligned}
f_{1}(t)= & \mathcal{N}_{1}(v)-\frac{v(t)}{\theta} \widehat{\mathcal{N}_{1}}(v)(t, 0) \\
& +\frac{1}{h_{1}(t)}\left(\mathcal{N}_{2}(v)-\frac{v(t)}{\theta} \widehat{\mathcal{N}_{2}}(v)(t, 0)\right) .
\end{aligned}
$$

In the case $a_{0} \equiv 0$, we denote $e^{2 \varphi(t)}=h_{2}(t)$ and obtain the following system of integral equations:

$$
\begin{gathered}
r=-\int_{0}^{t} h_{2}^{-1 / 2}(\tau) \mathscr{G}(t-\tau) f_{2}(\tau) d \tau, \\
h_{2}=1+\frac{1}{2 \theta} \int_{0}^{t}\left(\sqrt{h_{2}(\tau)} \widehat{\mathcal{\aleph}_{1}}(v)+\widehat{\mathcal{N}_{2}}(v)\right)(\tau, 0) d \tau,
\end{gathered}
$$


where

$$
f_{2}(t)=\mathcal{N}_{1}(v)-\frac{v(t)}{\theta} \widehat{\mathcal{N}}_{1}(v)(t, 0)+\frac{1}{\sqrt{h_{2}(t)}}\left(\mathcal{N}_{2}(v)-\frac{v(t)}{\theta} \widehat{\mathcal{N}}_{2}(v)(t, 0)\right) .
$$

Denote

$$
\begin{aligned}
& \mathscr{A}_{j}\left(r, h_{j}\right)(t)=-\int_{0}^{t} h_{j}^{-1 / j}(\tau) \mathscr{G}(t-\tau) f_{j}(\tau) d \tau, \quad j=1,2, \\
& \mathscr{B}_{1}\left(r, h_{1}\right)(t)=1+\frac{1}{\theta} \int_{0}^{t}\left(\widehat{\mathcal{N}_{1}}(v)+\frac{1}{h_{1}(\tau)} \widehat{\mathcal{N}_{2}}(v)\right)(\tau, 0) d \tau
\end{aligned}
$$

in the case $a_{0} \neq 0$ and

$$
\mathscr{B}_{2}\left(r, h_{2}\right)(t)=1+\frac{1}{2 \theta} \int_{0}^{t}\left(\sqrt{h_{2}(\tau)}{\widehat{\aleph_{1}}}_{1}(v)+\widehat{\mathcal{N}_{2}}(v)\right)(\tau, 0) d \tau
$$

in the case $a_{0} \equiv 0$. We prove that $\left(\mathscr{A}_{j}, \mathscr{B}_{j}\right)$ is the contraction mapping in the set

$$
\begin{aligned}
\mathbf{X}=\left\{r \in \mathbf{C}\left((0, \infty) ; \mathbf{D}^{\alpha, \sigma}\right), h_{j} \in \mathbf{C}((0, \infty)):\right. \\
\left.\quad\|g(t) r(t)\|_{\mathbf{X}} \leq C \varepsilon^{2}, \frac{1}{2} g(t) \leq h_{j}(t) \leq 2 g(t) \forall t>0\right\},
\end{aligned}
$$

where

$$
\begin{aligned}
\|\phi\|_{\mathbf{X}}= & \sup _{\rho \in[-\gamma, \alpha+\gamma]} \sup _{t>0}\langle t\rangle^{(\rho+1) / \delta}\|\phi(t)\|_{\mathrm{A}^{\rho, 1}} \\
& +\sup _{\rho \in[0, \alpha+\gamma]} \sup _{t>0}\langle t\rangle^{\rho / \delta}\|\phi(t)\|_{\mathbf{A}^{\rho, \infty}} \\
& +\sup _{s \in[0, \sigma]} \sup _{1 \leq p \leq \infty} \sup _{t>0}\{t\}^{s / v}\langle t\rangle^{1+\gamma / \delta+1 / \delta p}\|\phi(t)\|_{\mathbf{B}^{s, p}} \\
& +\sup _{\rho=0, \alpha, \beta} \sup _{s \in[0, \sigma]} \sup _{t>0}\{t\}^{s / v}\langle t\rangle^{(\rho-\gamma) / \delta}\|\phi(t)\|_{\mathbf{D}^{\rho, s}}
\end{aligned}
$$

here $\gamma \in(0, \min (1, \delta))$ is such that $\gamma<\alpha$ if $\alpha>0$ and $\gamma<\beta$ if $\beta>0$. First we prove that the mapping $\left(\mathscr{A}_{j}, \mathscr{B}_{j}\right)$ transforms the set $\mathbf{X}$ into itself. When $\left(r, h_{j}\right) \in \mathbf{X}$, we get by Lemma 2.4

$$
\left\|\mathcal{N}_{1}(v)+h_{j}^{-1 / j} \mathcal{N}_{2}(v)\right\|_{\mathbf{Y}} \leq C \varepsilon^{2}, \quad\left\|\frac{v(\tau)}{\theta} \hat{\mathcal{N}}(v)(\tau, 0)\right\|_{\mathbf{Y}} \leq C \varepsilon^{2}
$$

where the norm

$$
\begin{aligned}
\|\phi\|_{\mathbf{Y}}= & \sup _{1 \leq p \leq \infty} \sup _{t>0}\langle t\rangle^{1+1 / \delta p}\|\phi(t)\|_{\mathbf{A}^{0, p}} \\
& +\sup _{1 \leq p \leq \infty} \sup _{t>0}\langle t\rangle^{1+\gamma / \delta+1 / \delta p}\{t\}^{\sigma / v}\|\phi(t)\|_{\mathbf{B}^{0, p}} \\
& +\sup _{t>0}\langle t\rangle^{1-\gamma / \delta}\{t\}^{\sigma / v}\|\phi(t)\|_{\mathbf{D}^{0,0}} .
\end{aligned}
$$


Hence,

$$
\begin{aligned}
& \left\|f_{1}\right\|_{\mathbf{Y}}=\left\|\mathcal{N}_{1}(v)-\frac{v(t)}{\theta} \widehat{\mathcal{N}_{1}}(v)(t, 0)+\frac{1}{h_{1}(t)}\left(\mathcal{N}_{2}(v)-\frac{v(t)}{\theta} \widehat{\mathcal{N}_{2}}(v)(t, 0)\right)\right\|_{\mathbf{Y}} \leq C \varepsilon^{2}, \\
& \left\|f_{2}\right\|_{\mathbf{Y}}=\left\|\mathcal{N}_{1}(v)-\frac{v(t)}{\theta} \widehat{\widehat{N}_{1}}(v)(t, 0)+\frac{1}{\sqrt{h_{2}(t)}}\left(\mathcal{N}_{2}(v)-\frac{v(t)}{\theta} \widehat{\mathcal{N}_{2}}(v)(t, 0)\right)\right\|_{\mathbf{Y}} \leq C \varepsilon^{2} .
\end{aligned}
$$

Therefore, applying Lemma 2.3, we get the estimates

$$
\left\|g(t) \mathscr{A}_{j}\left(r, h_{j}\right)(t)\right\|_{\mathbf{X}} \leq C\left\|g(t) \int_{0}^{t} h_{j}^{-1}(\tau) \mathscr{G}(t-\tau) f_{j}(\tau) d \tau\right\|_{\mathbf{X}} \leq C\left\|f_{j}\right\|_{\mathbf{Y}} \leq C \varepsilon^{2}
$$

Furthermore, when $r \in \mathrm{X}$, we have the estimates $\|v\|_{\mathrm{X}} \leq C \varepsilon$ and $\left\|g\left(v-\mathscr{G}(t) u_{0}\right)\right\|_{\mathrm{X}} \leq$ $C\|g r\|_{\mathrm{X}} \leq C \varepsilon^{2}$. Via Lemma 2.5, we obtain (2.66) if $a_{0} \neq 0$ and (2.67) if $a_{0} \equiv 0$. Hence,

$$
\frac{1}{2} g(t) \leq h_{j}(t) \leq 2 g(t)
$$

for all $t>0$. Thus, $\left(\mathscr{A}_{j}, \mathscr{B}_{j}\right)$ transforms the set $\mathbf{X}$ into itself.

In the same manner, we consider the differences $\mathscr{A}_{j}\left(r, h_{j}\right)-\mathscr{A}_{j}\left(\tilde{r}, \tilde{h}_{j}\right)$ and $\mathscr{B}_{j}\left(r, h_{j}\right)-$ $\mathscr{B}_{j}\left(\tilde{r}, \tilde{h}_{j}\right)$ to see that the transformation $\left(\mathscr{A}_{j}, \mathscr{B}_{j}\right)$ is the contraction mapping. Therefore, there exists a unique solution $\left(r, h_{j}\right)$ of the system of integral equations (3.8) in the space $\mathbf{X}$. From Lemma 2.5, we see that

$$
h_{j}(t)=\varkappa \log t+O(\log \log t)
$$

for $t \rightarrow \infty$. Therefore, via formulas $u(t, x)=e^{-\varphi(t)} v(t, x)=e^{-\varphi(t)}\left(\varphi v_{0}+r\right)$, we obtain the asymptotic formula of the theorem. Theorem 1.1 is proved.

ACKNOWLEDGMENTS. The work of Elena I. Kaikina and Pavel I. Naumkin is partially supported by CONACYT. The authors are grateful to the unknown referees for many useful suggestions and comments.

\section{REFERENCES}

[1] M. J. Ablowitz and H. Segur, Solitons and the Inverse Scattering Transform, SIAM Studies in Applied Mathematics, vol. 4, Society for Industrial and Applied Mathematics (SIAM), Philadelphia, 1981.

[2] R. E. Cardiel and P. I. Naumkin, Asymptotics for nonlinear dissipative equations in the super critical case, Mathematical Results in Quantum Mechanics (Taxco, 2001), Contemp. Math., vol. 307, American Mathematical Society, Rhode Island, 2002, pp. 47-67.

[3] P. A. Deift, A. R. Its, and X. Zhou, Long-time asymptotics for integrable nonlinear wave equations, Important Developments in Soliton Theory, Springer Ser. Nonlinear Dynam., Springer-Verlag, Berlin, 1993, pp. 181-204.

[4] D. B. Dix, Large-Time Behavior of Solutions of Linear Dispersive Equations, Lecture Notes in Mathematics, vol. 1668, Springer-Verlag, Berlin, 1997.

[5] M. Escobedo and O. Kavian, Asymptotic behavior of positive solutions of a non-linear heat equation, Houston J. Math. 13 (1987), no. 4, 39-50.

[6] M. Escobedo, O. Kavian, and H. Matano, Large time behavior of solutions of a dissipative semilinear heat equation, Comm. Partial Differential Equations 20 (1995), no. 7-8, 1427-1452. 
[7] V. A. Galaktionov, S. P. Kurdyumov, and A. A. Samarskii, On asymptotic eigenfunctions of the Cauchy problem for a nonlinear parabolic equation, Math. USSR-Sb. 54 (1986), 421-455.

[8] V. A. Galaktionov and J. L. Vazquez, Asymptotic behavior of nonlinear parabolic equations with critical exponents. A dynamical systems approach, J. Funct. Anal. 100 (1991), no. 2, 435-462.

[9] A. Gmira and L. Véron, Large time behaviour of the solutions of a semilinear parabolic equation in $\mathbf{R}^{N}$, J. Differential Equations 53 (1984), no. 2, 258-276.

[10] N. Hayashi, N. Ito, E. I. Kaikina, and P. I. Naumkin, Large time decay of solutions to Burgers type equations, preprint, 2002.

[11]__ On some nonlinear dissipative equations with sub-critical nonlinearities, preprint, 2002.

[12] N. Hayashi, E. I. Kaikina, and P. I. Naumkin, Large-time behaviour of solutions to the dissipative nonlinear Schrödinger equation, Proc. Roy. Soc. Edinburgh Sect. A 130 (2000), no. 5, 1029-1043.

[13] _ Large time behavior of solutions to the Landau-Ginzburg type equations, Funkcial. Ekvac. 44 (2001), no. 1, 171-200.

[14] _ Asymptotic expansion of small solutions to the Landau-Ginzburg type equations, Asymptot. Anal. 32 (2002), no. 2, 91-106.

[15]__ Asymptotics in the critical case for Whitham type equations, preprint, 2002.

[16] _ Global existence and time decay of small solutions to the Landau-Ginzburg type equations, J. Anal. Math. 90 (2003), 141-173.

[17] Landau-Ginzburg type equations in the subcritical case, Commun. Contemp. Math. 5 (2003), no. 1, 127-145.

[18] S. Kamin and L. A. Peletier, Large time behaviour of solutions of the porous media equation with absorption, Israel J. Math. 55 (1986), no. 2, 129-146.

[19] O. Kavian, Remarks on the large time behaviour of a nonlinear diffusion equation, Ann. Inst. H. Poincaré Anal. Non Linéaire 4 (1987), no. 5, 423-452.

[20] P. I. Naumkin and I. A. Shishmarëv, Nonlinear Nonlocal Equations in the Theory of Waves, Translations of Mathematical Monographs, vol. 133, American Mathematical Society, Rhode Island, 1994.

[21] G. B. Whitham, Linear and Nonlinear Waves, John Wiley \& Sons, New York, 1974.

[22] E. Zuazua, Some recent results on the large time behavior for scalar parabolic conservation laws, Elliptic and Parabolic Problems (Pont-à-Mousson, 1994), Pitman Res. Notes Math. Ser., vol. 325, Longman Sci. Tech., Harlow, 1995, pp. 251-263.

Nakao Hayashi: Department of Mathematics, Graduate School of Science, Osaka University, Toyonaka, Osaka 560-0043, Japan

E-mail address: nhayashi@math . wani . osaka-u .ac.jp

Elena I. Kaikina: Departamento de Ciencias Básicas, Instituto Tecnológico de Morelia, Morelia CP 58120, Michoacán, Mexico

E-mail address: ekaikina@elec.itmorelia.edu.mx

Pavel I. Naumkin: Instituto de Matemáticas, Universidad Nacional Autonoma de México (UNAM), Campus Morelia, AP 61-3 (Xangari), Morelia CP 58089, Michoacán, Mexico

E-mail address: pave1ni@matmor.unam.mx 


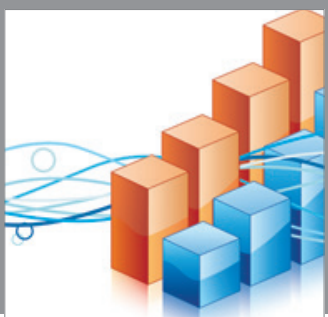

Advances in

Operations Research

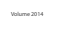

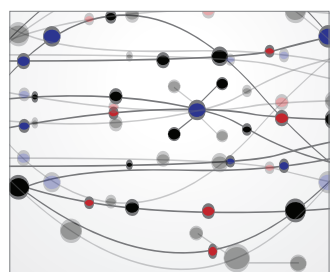

\section{The Scientific} World Journal
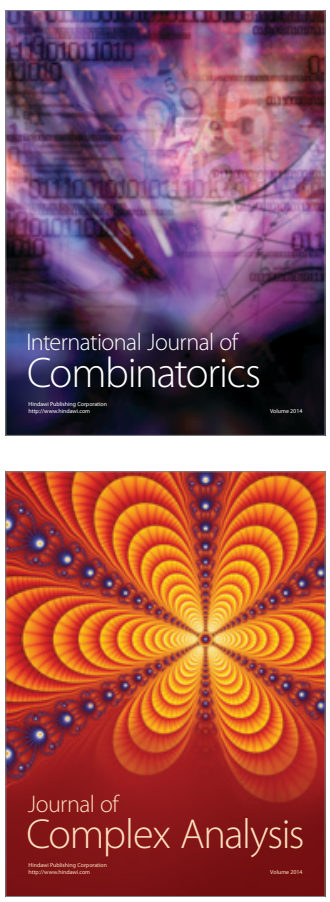

International Journal of

Mathematics and

Mathematical

Sciences
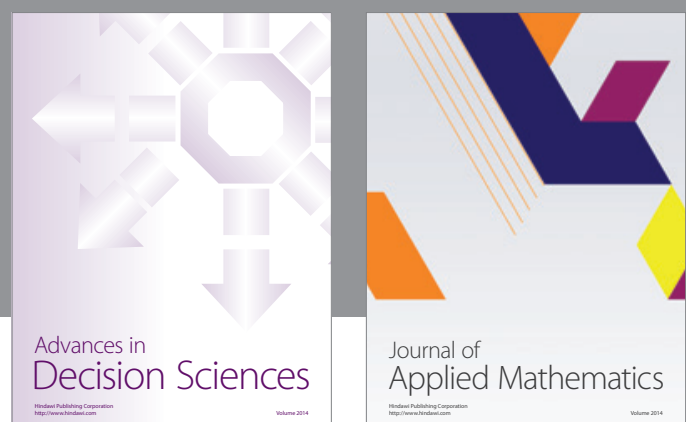

Journal of

Applied Mathematics
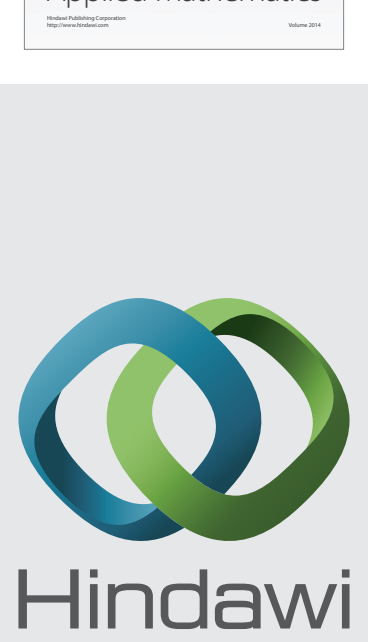

Submit your manuscripts at http://www.hindawi.com
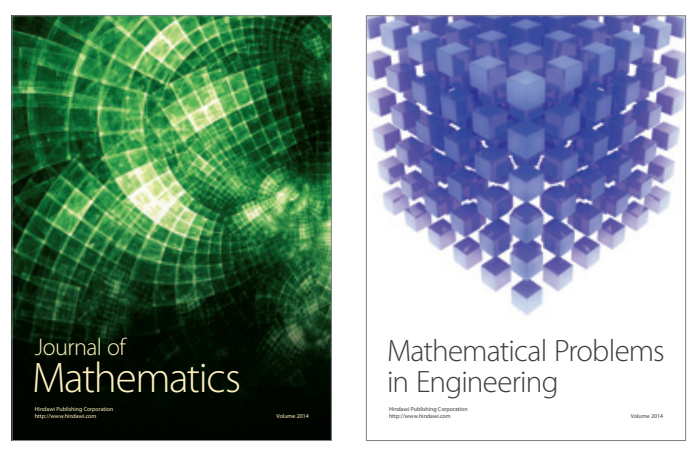

Mathematical Problems in Engineering
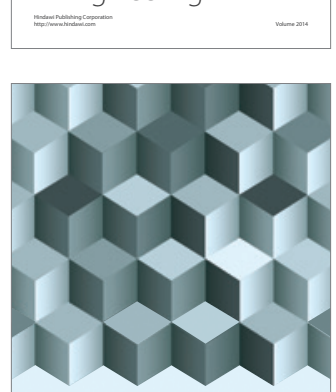

Journal of

Function Spaces
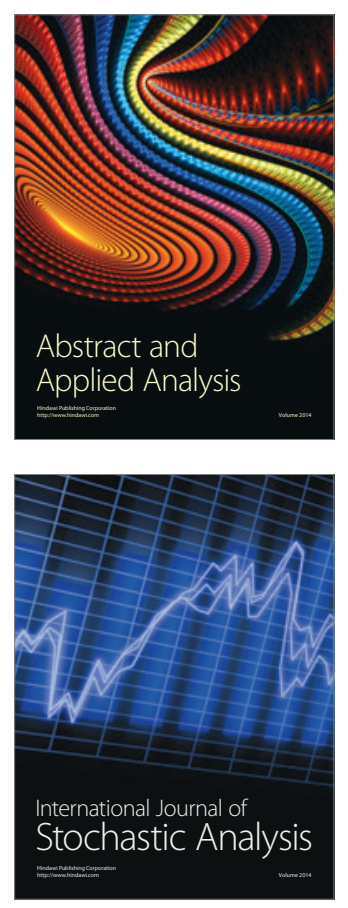

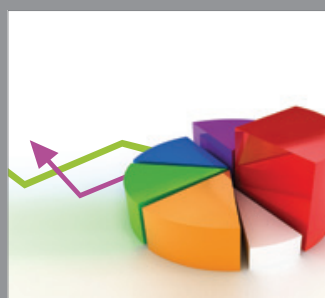

ournal of

Probability and Statistics

Promensencen
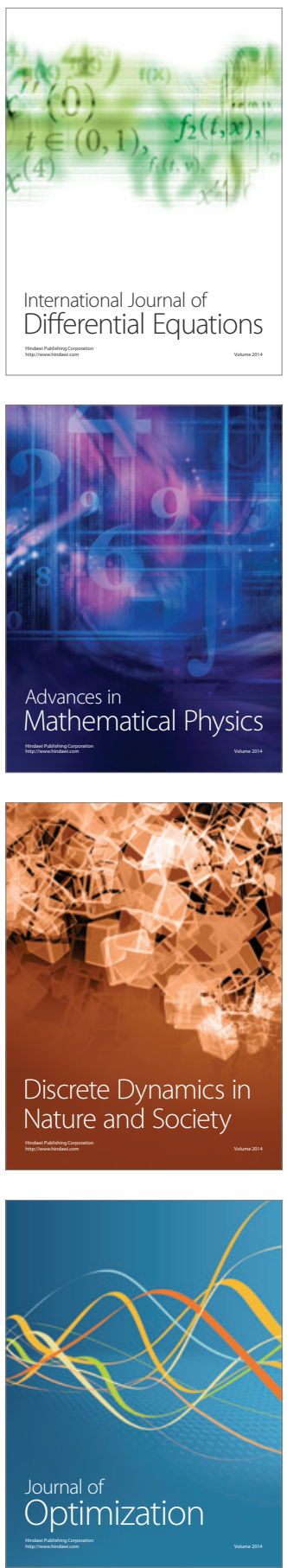